Article

\title{
Polynomial Decay Rate for Kirchhoff Type in Viscoelasticity with Logarithmic Nonlinearity and Not Necessarily Decreasing Kernel
}

\author{
Salah Boulaaras ${ }^{1,2, *(\mathbb{D})}$, Alaeddin Draifia ${ }^{3}$ and Mohammad Alnegga ${ }^{1}$ (i) \\ 1 Department of Mathematics, College of Sciences and Arts, Al-Rass, Qassim University, Buraydah 51921, \\ Saudi Arabia; m7mmad_111@hotmail.com \\ 2 Laboratory of Fundamental and Applied Mathematics of Oran (LMFAO), University of Oran 1, \\ Ahmed Benbella, Oran 31000, Algeria \\ 3 Laboratory of Mathematics, Informatics and Systems (LAMIS), Larbi Tebessi University, Tebessa 12002, \\ Algeria; draifia1991@gmail.com \\ * Correspondence: saleh_boulaares@yahoo.fror S.Boularas@qu.edu.sa
}

Received: 14 January 2019; Accepted: 12 February 2019; Published: 14 February 2019

check for updates

\begin{abstract}
This paper describes a polynomial decay rate of the solution of the Kirchhoff type in viscoelasticity with logarithmic nonlinearity, where an asymptotically-stable result of the global solution is obtained taking into account that the kernel is not necessarily decreasing.
\end{abstract}

Keywords: global existence; polynomial decay; viscoelastic damping; intrinsic decay rates

\section{Introduction}

Consider the quasilinear viscoelastic problem:

$$
\begin{gathered}
\left|u_{t}\right|^{\rho} u_{t t}+M(t) \Delta^{2} u(t)+\Delta^{2} u_{t t}(t) \\
-\int_{0}^{t} h(t-s) \Delta^{2} u(s) d s=u|u|^{\gamma-2} \ln |u|^{k}, \quad \text { in } \Omega \times(0, \infty), \\
u(x, t)=\frac{\partial u}{\partial v}(x, t)=0 \text { in } \partial \Omega \times(0, \infty), \\
u(x, 0)=u^{0}, u_{t}(x, 0)=u^{1} \text { for } x \in \Omega
\end{gathered}
$$

with:

$$
M(t):=\left(\xi_{0}+\xi_{1}\|\Delta u(t)\|_{L^{2}(\Omega)}^{2 \beta}\right), \beta \geq 1,
$$

where $\Omega \subset \mathbb{R}^{N}$ is a bounded domain with a sufficiently smooth boundary $\partial \Omega, \gamma \geq 2, k, \xi_{0}$ and $\xi_{1}$ are positive constants, $\rho \geq 0$ for $N=1,2$ and $0 \leq \rho \leq \frac{4}{N-2}$ for $N \geq 3$, and $h$ is a relaxation function, which will be specified later. From the physical point of view, the problem (1)-(3) is related to the spillover problem with memory and the panel flutter equation. Viscoelasticity has an important role in the study of biological phenomena, and it can have a strong effect on some factors that have a correlation with biological phenomenain general. A viscoelastic material will return back to its original size after an influential force has been detached, even though it will remain for a specific time in its original shape; see [1-11].

Recently, viscoelasticity problems have been handled carefully in several papers, and other results relating the global existence and decay of the global solution have been found (see [1-7,10-23]), where the function of the relaxation was supposed to be either exponential decay or polynomial decay. In [7], the mathematicians searched for a viscoelastic equation for more general decaying kernels 
and obtained some general decay results, from which the common exponential or polynomial rates were more specific samples of them. Later, many papers, using almost identical techniques, achieved analogous general decay outcomes. See $[11,24]$. Our decay rate obtained in the third section is less general than that obtained in $[7,8]$, where a common decay rate outcome was established in order to let the functions of the relaxation satisfy:

$$
g^{\prime}(t) \leq-H(g(t)), t \geq 0, H(0)=0
$$

a positive function $H \in C^{1}\left(\mathbb{R}^{+}\right)$, where $H$ is either linear or a strictly-increasing and strictly-convex $C^{2}$ function on $(0, r], 1>r$. In [7], the exponential decay for the viscoelastic wave equation of the solution with an unnecessarily decreasing kernel was handled. More specifically, the mathematicians had an exponential decay outcome as time tended to infinity $h^{\prime}(t)+\gamma h(t) \geq 0$ for all $t \geq 0$ provided that $\left(h^{\prime}(t)+\gamma h(t)\right) e^{\alpha t} \in L^{1}(0, \infty)$ for some $\alpha>0$. Their proof depended on another technique, the so-called "Lyapunov functional". In this present work, we follow up with the same steps of the previous result in $[7,10]$ for a new class of Kirchhoff hyperbolic equations on bounded domain polynomial decay of the Kirchhoff type in viscoelasticity combined with the right-hand side defined as a logarithmic nonlinearity, and the kernel is not necessarily decreasing taking into account that the similar conditions in the last ones in $([7,10])$ are considered. The logarithmic nonlinearity is of much use in physics, since it naturally appears in inflation cosmology and supersymmetric filed theories, quantum mechanics, and nuclear physics (see $[5,14])$. This kind of problem can be applied in many different areas of physics such as nuclear physics, optics, and geophysics (see [3,15]).

The outline of the paper is as follows. In the second section, we give some basic concepts related to our problem given by (1)-(3). In Section 3, we prove our principle result.

\section{Preliminaries and Assumptions}

We use the standard Lebesgue space $L^{2}(\Omega)$ and Sobolev space $H_{0}^{1}(\Omega)$. For a Hilbert space $X$, we denote $(. . .)_{X}$ and $\|.\|_{X}$, the inner product and norm of $X$, respectively. For simplicity, we denote $(.,)_{L^{2}(\Omega)}$ and $\|\cdot\|_{L^{2}(\Omega)}$ by $(.,$.$) and \|$.$\| , respectively.$

As in previous work in $([7,10])$, we impose the following conditions on the relaxation function $h$. Namely, we suppose that the kernel $h(t)$ is a $C^{1}\left(\mathbb{R}_{+}, \mathbb{R}_{+}\right)$function satisfying

(A1) $\int_{0}^{\infty} h(s) d s<\xi_{0}$.

(A2) $\int_{0}^{\infty} h^{1-\tau}(s) d s<\infty$, for $0<\tau<2-r<1$ where $1<r<\frac{3}{2}$.

(A3) There exists a positive differentiable function $\xi(t)$ such that:

$$
h^{\prime}(t)+\xi(t) h^{r}(t) \geq 0
$$

and:

$$
e^{\alpha t}\left[h^{\prime}(t)+\xi(t) h^{r}(t)\right] \in L^{1}\left(\mathbb{R}_{+}\right),
$$

where $\left(1 \leq r<\frac{3}{2}\right)$ and for $\alpha>0$, and $\xi(t)$ satisfies some positive constant $L$,

$$
\left|\frac{\xi^{\prime}(t)}{\xi(t)}\right| \leq L, \quad \xi^{\prime}(t) \leq 0, \quad \int_{0}^{\infty} \xi(s) d s=\infty, \quad t \geq 0
$$

Furthermore, where $1<r<\frac{3}{2}$ for any $t \geq 0$, there exists a positive constant $C_{r}$ depending only on $r$, such that:

$$
\frac{t}{\left(1+\int_{0}^{t} \xi(s) d s\right)^{\frac{1}{2(r-1)}}} \leq C_{r}, \forall t \geq 0 .
$$


Notation: We denote by $l, \bar{l}, l_{\alpha}, \bar{l}_{\alpha}$ and $h$ the following expressions:

$$
\left\{\begin{array}{l}
\xi(t) l(t):=h^{\prime}(t)+\xi(t) h^{r}(t), \\
\bar{l}:=\int_{0}^{\infty} l(s) d s, \quad l_{\alpha}:=e^{\alpha t} l(t), \\
\bar{l}_{\alpha}:=\int_{0}^{\infty} l_{\alpha}(s) d s, \quad \bar{h}:=\int_{0}^{\infty} h(s) d s .
\end{array}\right.
$$

and we recall the binary notation:

$$
(h \square w)(t):=\int_{0}^{t} h(t-s)\|w(x, s)-w(x, t)\|_{L^{2}(\Omega)}^{2} d s .
$$

Lemma 1. The classical energy associated with (1)-(3) is defined by:

$$
\begin{aligned}
E(t): & =\frac{1}{\rho+2}\left\|u_{t}(t)\right\|_{\rho+2}^{\rho+2}+\frac{1}{2}\left(\xi_{0}+\frac{\xi_{1}}{(\beta+1)}\|\Delta u(t)\|_{L^{2}(\Omega)}^{2 \beta}\right)\|\Delta u(t)\|_{L^{2}(\Omega)}^{2} \\
& +\frac{1}{2}\left\|\Delta u_{t}(t)\right\|_{L^{2}(\Omega)}^{2}-\frac{1}{\gamma}\left(\int_{\Omega}|u(t)|^{\gamma} \ln |u|^{k} d x\right)+\frac{k}{\gamma^{2}}\|u(t)\|_{\gamma}^{\gamma}
\end{aligned}
$$

and its derivative is:

$$
\frac{d}{d t}\{E(t)\}=\int_{0}^{t} h(t-s)\left(\Delta u(s), \Delta u_{t}(t)\right)_{L^{2}(\Omega)} d s
$$

Proof. Multiplying (1) by $u_{t}$ and by integration over $(\Omega)$, we have:

$$
\begin{aligned}
& \left(\left|u_{t}\right|^{\rho} u_{t t}(t), u_{t}(t)\right)_{L^{2}(\Omega)}+\left(M(t) \Delta^{2} u(t), u_{t}(t)\right)_{L^{2}(\Omega)} \\
& +\left(\Delta^{2} u_{t t}(t), u_{t}(t)\right)_{L^{2}(\Omega)}-\left(\int_{0}^{t} h(t-s) \Delta^{2} u(s) d s, u_{t}(t)\right)_{L^{2}(\Omega)} \\
= & \left(u|u|^{\gamma-2} \ln |u|^{k}, u_{t}(t)\right)_{L^{2}(\Omega)}
\end{aligned}
$$

By using:

$$
u_{t t}(t) u_{t}(t)=\frac{1}{2} \frac{d}{d t}\left\{u_{t}^{2}(t)\right\}
$$

we get by direct calculation:

$$
\begin{aligned}
\left(\left|u_{t}\right|^{\rho} u_{t t}(t), u_{t}(t)\right)_{L^{2}(\Omega)} & =\int_{\Omega}\left|u_{t}\right|^{\rho} \frac{1}{2} \frac{d}{d t}\left\{u_{t}^{2}(t)\right\} d x \\
& =\frac{1}{2} \int_{\Omega} \frac{d}{d t}\left\{\left|u_{t}\right|^{\rho} u_{t}^{2}(t)\right\} d x-\frac{1}{2} \int_{\Omega} \frac{d}{d t}\left\{\left|u_{t}\right|^{\rho}\right\} u_{t}^{2}(t) d x \\
& =\frac{1}{2} \frac{d}{d t}\left\{\int_{\Omega}\left|u_{t}\right|^{\rho+2} d x\right\}-\frac{\rho}{2} \int_{\Omega}\left|u_{t}\right|^{\rho-2} u_{t t}(t) u_{t}(t) u_{t}^{2}(t) d x \\
& =\frac{1}{2} \frac{d}{d t}\left\{\left\|u_{t}(t)\right\|_{\rho+2}^{\rho+2}\right\}-\frac{\rho}{2}\left(\left|u_{t}\right|^{\rho} u_{t t}(t), u_{t}(t)\right)_{L^{2}(\Omega)},
\end{aligned}
$$

then:

$$
\left(\left|u_{t}\right|^{\rho} u_{t t}(t), u_{t}(t)\right)_{L^{2}(\Omega)}=\frac{1}{\rho+2} \frac{d}{d t}\left\{\left\|u_{t}(t)\right\|_{\rho+2}^{\rho+2}\right\} .
$$


By using integration by parts, we have:

$$
\begin{aligned}
& \left(M(t) \Delta^{2} u(t), u_{t}(t)\right)_{L^{2}(\Omega)} \\
= & \left(\xi_{0}+\xi_{1}\|\Delta u(t)\|_{L^{2}(\Omega)}^{2 \beta} \int_{\Omega} \Delta^{2} u(t) u_{t}(t) d x\right. \\
= & \left(\xi_{0}+\xi_{1}\|\Delta u(t)\|_{L^{2}(\Omega)}^{2 \beta}\right) \int_{\Omega} \Delta u(t) \Delta u_{t}(t) d x \\
= & \left(\xi_{0}+\xi_{1}\|\Delta u(t)\|_{L^{2}(\Omega)}^{2 \beta} \frac{1}{2} \frac{d}{d t}\left\{\int_{\Omega}|\Delta u(t)|^{2} d x\right\}\right. \\
= & \frac{\xi_{0}}{2} \frac{d}{d t}\left\{\|\Delta u(t)\|_{L^{2}(\Omega)}^{2}\right\}+\frac{\xi_{1}}{2}\|\Delta u(t)\|_{L^{2}(\Omega)}^{2 \beta} \frac{d}{d t}\left\{\|\Delta u(t)\|_{L^{2}(\Omega)}^{2}\right\} \\
= & \frac{\xi_{0}}{2} \frac{d}{d t}\left\{\|\Delta u(t)\|_{L^{2}(\Omega)}^{2}\right\}+\frac{\xi_{1}}{2(\beta+1)} \frac{d}{d t}\left\{\|\Delta u(t)\|_{L^{2}(\Omega)}^{2(\beta+1)}\right\} \\
= & \frac{d}{d t}\left\{\frac{1}{2}\left(\xi_{0}+\frac{\xi_{1}}{(\beta+1)}\|\Delta u(t)\|_{L^{2}(\Omega)}^{2 \beta}\right)\|\Delta u(t)\|_{L^{2}(\Omega)}^{2}\right\} .
\end{aligned}
$$

Integrating by parts, we have:

$$
\begin{aligned}
\left(\Delta^{2} u_{t t}(t), u_{t}(t)\right)_{L^{2}(\Omega)} & =\left(\Delta u_{t t}(t), \Delta u_{t}(t)\right)_{L^{2}(\Omega)} \\
& =\frac{1}{2} \frac{d}{d t}\left\{\left\|\Delta u_{t}(t)\right\|_{L^{2}(\Omega)}^{2}\right\} .
\end{aligned}
$$

Also integrating by parts, we get:

$$
\begin{aligned}
& -\left(\int_{0}^{t} h(t-s) \Delta^{2} u(s) d s, u_{t}(t)\right)_{L^{2}(\Omega)} \\
= & -\int_{0}^{t} h(t-s)\left(\Delta u(s), \Delta u_{t}(t)\right)_{L^{2}(\Omega)} d s .
\end{aligned}
$$

By using:

$$
|u|^{\gamma-2} u(t) u_{t}(t)=\frac{1}{\gamma} \frac{d}{d t}\left\{|u(t)|^{\gamma}\right\},
$$

we get:

$$
\begin{aligned}
& \left(u|u|^{\gamma-2} \ln |u|^{k}, u_{t}(t)\right)_{L^{2}(\Omega)} \\
= & \int_{\Omega} \frac{1}{\gamma} \frac{d}{d t}\left\{|u(t)|^{\gamma}\right\} \ln |u|^{k} d x \\
= & \frac{1}{\gamma} \int_{\Omega} \frac{d}{d t}\left\{|u(t)|^{\gamma} \ln |u|^{k}\right\} d x-\frac{1}{\gamma} \int_{\Omega}|u(t)|^{\gamma} \frac{d}{d t}\left\{\ln |u|^{k}\right\} d x \\
= & \frac{1}{\gamma} \frac{d}{d t}\left\{\int_{\Omega}|u(t)|^{\gamma} \ln |u|^{k} d x\right\}-\frac{k}{\gamma} \int_{\Omega}|u(t)|^{\gamma-2} u(t) u_{t}(t) d x \\
= & \frac{1}{\gamma} \frac{d}{d t}\left\{\int_{\Omega}|u(t)|^{\gamma} \ln |u|^{k} d x\right\}-\frac{k}{\gamma^{2}} \int_{\Omega} \frac{d}{d t}\left\{|u(t)|^{\gamma}\right\} d x \\
= & \frac{d}{d t}\left\{\frac{1}{\gamma}\left(\int_{\Omega}|u(t)|^{\gamma} \ln |u|^{k} d x\right)-\frac{k}{\gamma^{2}}\|u(t)\|_{\gamma}^{\gamma}\right\} .
\end{aligned}
$$

By replacing (12)-(16) in (11), we get:

$$
\begin{aligned}
& \frac{1}{\rho+2} \frac{d}{d t}\left\{\left\|u_{t}(t)\right\|_{\rho+2}^{\rho+2}\right\} \\
& +\frac{d}{d t}\left\{\frac{1}{2}\left(\xi_{0}+\frac{\tilde{\xi}_{1}}{(\beta+1)}\|\Delta u(t)\|_{L^{2}(\Omega)}^{2 \beta}\right)\|\Delta u(t)\|_{L^{2}(\Omega)}^{2}\right\} \\
& +\frac{1}{2} \frac{d}{d t}\left\{\left\|\Delta u_{t}(t)\right\|_{L^{2}(\Omega)}^{2}\right\} \\
& -\int_{0}^{t} h(t-s)\left(\Delta u(s), \Delta u_{t}(t)\right)_{L^{2}(\Omega)} d s \\
= & \frac{d}{d t}\left\{\frac{1}{\gamma}\left(\int_{\Omega}|u(t)|^{\gamma} \ln |u|^{k} d x\right)-\frac{k}{\gamma^{2}}\|u(t)\|_{\gamma}^{\gamma}\right\},
\end{aligned}
$$

then (17) is equivalent to:

$$
\begin{aligned}
& \frac{d}{d t}\left\{\frac{1}{\rho+2}\left\|u_{t}(t)\right\|_{\rho+2}^{\rho+2}+\frac{1}{2}\left(\xi_{0}+\frac{\xi_{1}}{(\beta+1)}\|\Delta u(t)\|_{L^{2}(\Omega)}^{2 \beta}\right)\|\Delta u(t)\|_{L^{2}(\Omega)}^{2}\right. \\
& \left.+\frac{1}{2}\left\|\Delta u_{t}(t)\right\|_{L^{2}(\Omega)}^{2}-\frac{1}{\gamma}\left(\int_{\Omega}|u(t)|^{\gamma} \ln |u|^{k} d x\right)+\frac{k}{\gamma^{2}}\|u(t)\|_{\gamma}^{\gamma}\right\} \\
= & \int_{0}^{t} h(t-s)\left(\Delta u(s), \Delta u_{t}(t)\right)_{L^{2}(\Omega)} d s,
\end{aligned}
$$

and by using (9) in (18), we get (10) . 
The proof is complete.

Lemma 2. The modified energy for (1)-(3) is defined by:

$$
\begin{aligned}
e(t): & =\frac{1}{\rho+2}\left\|u_{t}(t)\right\|_{\rho+2}^{\rho+2}+\frac{1}{2}\left(\xi_{0}-\int_{0}^{t} h(s) d s\right)\|\Delta u(t)\|_{L^{2}(\Omega)}^{2} \\
& +\frac{\xi 1}{2(\beta+1)}\|\Delta u(t)\|_{L^{2}(\Omega)}^{2(\beta+1)}+\frac{1}{2}\left\|\Delta u_{t}(t)\right\|_{L^{2}(\Omega)}^{2} \\
& -\frac{1}{\gamma}\left(\int_{\Omega}|u(t)|^{\gamma} \ln |u|^{k} d x\right)+\frac{k}{\gamma^{2}}\|u(t)\|_{\gamma}^{\gamma}+\frac{1}{2}(h \square \Delta u)(t) .
\end{aligned}
$$

and its derivative satisfies the following:

$$
\begin{aligned}
\frac{d}{d t}\{e(t)\} & =\frac{1}{2}\left(h^{\prime} \square \Delta u\right)(t)-\frac{1}{2} h(t)\|\Delta u(t)\|_{L^{2}(\Omega)}^{2} \\
& \leq 0 .
\end{aligned}
$$

Proof. Now, for the estimation terms, we have:

$$
\begin{aligned}
& -\int_{0}^{t} h(t-s)\left(\Delta u(s), \Delta u_{t}(t)\right)_{L^{2}(\Omega)} d s \\
= & -\int_{0}^{t} h(t-s)\left[\int_{\Omega} \Delta u(x, s) \Delta u_{t}(x, t) d x\right] d s,
\end{aligned}
$$

and using:

$$
-\Delta u(x, s) \Delta u_{t}(x, t)=\frac{1}{2} \frac{d}{d t}\left\{|\Delta u(x, s)-\Delta u(x, t)|^{2}\right\}-\frac{1}{2} \frac{d}{d t}\left\{|\Delta u(x, t)|^{2}\right\},
$$

then:

$$
\begin{aligned}
& -\int_{0}^{t} h(t-s)\left(\Delta u(s), \Delta u_{t}(t)\right)_{L^{2}(\Omega)} d s \\
= & \int_{0}^{t} h(t-s) \int_{\Omega}\left(\frac{1}{2} \frac{d}{d t}\left\{|\Delta u(x, s)-\Delta u(x, t)|^{2}\right\}\right) d x d s \\
& -\int_{0}^{t} h(t-s) \int_{\Omega}\left(\frac{1}{2} \frac{d}{d t}\left\{|\Delta u(x, t)|^{2}\right\}\right) d x d s \\
= & \frac{1}{2} \int_{0}^{t} h(t-s)\left(\frac{d}{d t}\left\{\int_{\Omega}|\Delta u(x, s)-\Delta u(x, t)|^{2} d x\right\}\right) d s \\
& -\frac{1}{2} \int_{0}^{t} h(t-s)\left(\frac{d}{d t}\left\{\|\Delta u(t)\|_{L^{2}(\Omega)}^{2}\right\}\right) d s .
\end{aligned}
$$

Using the direct account, we find:

$$
\begin{aligned}
& \frac{1}{2} \int_{0}^{t} h(t-s) \frac{d}{d t}\left\{\int_{\Omega}|\Delta u(x, s)-\Delta u(x, t)|^{2} d x\right\} d s \\
= & \frac{1}{2} \int_{0}^{t} \frac{d}{d t}\left\{h(t-s)\left(\int_{\Omega}|\Delta u(x, s)-\Delta u(x, t)|^{2} d x\right)\right\} d s \\
& -\frac{1}{2} \int_{0}^{t} h^{\prime}(t-s)\left(\int_{\Omega}|\Delta u(x, s)-\Delta u(x, t)|^{2} d x\right) d s \\
= & \frac{1}{2} \frac{d}{d t}\left\{\int_{0}^{t} h(t-s) \int_{\Omega}|\Delta u(x, s)-\Delta u(x, t)|^{2} d x d s\right\} \\
& -\frac{1}{2} \int_{0}^{t} h^{\prime}(t-s)\left(\int_{\Omega}|\Delta u(x, s)-\Delta u(x, t)|^{2} d x\right) d s \\
= & \frac{1}{2} \frac{d}{d t}\{(h \square \Delta u)(t)\}-\frac{1}{2}\left(h^{\prime} \square \Delta u\right)(t),
\end{aligned}
$$

and:

$$
\begin{aligned}
& -\frac{1}{2} \int_{0}^{t} h(t-s)\left(\frac{d}{d t}\left\{\|\Delta u(t)\|_{L^{2}(\Omega)}^{2}\right\}\right) d s \\
= & -\frac{1}{2}\left(\int_{0}^{t} h(t-s) d s\right)\left(\frac{d}{d t}\left\{\|\Delta u(t)\|_{L^{2}(\Omega)}^{2}\right\}\right) \\
= & -\frac{1}{2}\left(\int_{0}^{t} h(s) d s\right) \frac{d}{d t}\left\{\|\Delta u(t)\|_{L^{2}(\Omega)}^{2}\right\} \\
= & -\frac{1}{2} \frac{d}{d t}\left\{\left(\int_{0}^{t} h(s) d s\right)\|\Delta u(t)\|_{L^{2}(\Omega)}^{2}\right\}+\frac{1}{2} h(t)\|\Delta u(t)\|_{L^{2}(\Omega)}^{2} .
\end{aligned}
$$


By replacing (22) and (23) in (21), we get:

$$
\begin{aligned}
& -\int_{0}^{t} h(t-s)\left(\Delta u(s), \Delta u_{t}(t)\right)_{L^{2}(\Omega)} d s \\
= & \frac{1}{2} \frac{d}{d t}\{(h \square \Delta u)(t)\}-\frac{1}{2}\left(h^{\prime} \square \Delta u\right)(t) \\
& -\frac{1}{2} \frac{d}{d t}\left\{\left(\int_{0}^{t} h(s) d s\right)\|\Delta u(t)\|_{L^{2}(\Omega)}^{2}\right\}+\frac{1}{2} h(t)\|\Delta u(t)\|_{L^{2}(\Omega)}^{2} \\
= & \frac{d}{d t}\left\{\frac{1}{2}(h \square \Delta u)(t)-\frac{1}{2}\left(\int_{0}^{t} h(s) d s\right)\|\Delta u(t)\|_{L^{2}(\Omega)}^{2}\right\} \\
& -\frac{1}{2}\left(h^{\prime} \square \Delta u\right)(t)+\frac{1}{2} h(t)\|\Delta u(t)\|_{L^{2}(\Omega)}^{2} .
\end{aligned}
$$

By replacing (24) into (18), we get:

$$
\begin{aligned}
& \quad \frac{d}{d t}\left\{\frac{1}{\rho+2}\left\|u_{t}(t)\right\|_{\rho+2}^{\rho+2}+\frac{1}{2}\left(\xi_{0}+\frac{\xi_{1}}{(\beta+1)}\|\Delta u(t)\|_{L^{2}(\Omega)}^{2 \beta}\right)\|\Delta u(t)\|_{L^{2}(\Omega)}^{2}\right. \\
& \left.+\frac{1}{2}\left\|\Delta u_{t}(t)\right\|_{L^{2}(\Omega)}^{2}-\frac{1}{\gamma}\left(\int_{\Omega}|u(t)|^{\gamma} \ln |u|^{k} d x\right)+\frac{k}{\gamma^{2}}\|u(t)\|_{\gamma}^{\gamma}\right\} \\
& +\frac{d}{d t}\left\{\frac{1}{2}(h \square \Delta u)(t)-\frac{1}{2}\left(\int_{0}^{t} h(s) d s\right)\|\Delta u(t)\|_{L^{2}(\Omega)}^{2}\right\} \\
& -\frac{1}{2}\left(h^{\prime} \square \Delta u\right)(t)+\frac{1}{2} h(t)\|\Delta u(t)\|_{L^{2}(\Omega)}^{2} \\
& =0,
\end{aligned}
$$

then $(25)$ is equivalent to:

$$
\begin{aligned}
& \frac{d}{d t}\left\{\frac{1}{\rho+2}\left\|u_{t}(t)\right\|_{\rho+2}^{\rho+2}+\frac{1}{2}\left(\xi_{0}-\int_{0}^{t} h(s) d s\right)\|\Delta u(t)\|_{L^{2}(\Omega)}^{2}\right. \\
& +\frac{\xi_{1}}{2(\beta+1)}\|\Delta u(t)\|_{L^{2}(\Omega)}^{2(\beta+1)}+\frac{1}{2}\left\|\Delta u_{t}(t)\right\|_{L^{2}(\Omega)}^{2} \\
& \left.-\frac{1}{\gamma}\left(\int_{\Omega}|u(t)|^{\gamma} \ln |u|^{k} d x\right)+\frac{k}{\gamma^{2}}\|u(t)\|_{\gamma}^{\gamma}+\frac{1}{2}(h \square \Delta u)(t)\right\} \\
= & \frac{1}{2}\left(h^{\prime} \square \Delta u\right)(t)-\frac{1}{2} h(t)\|\Delta u(t)\|_{L^{2}(\Omega)}^{2},
\end{aligned}
$$

by using (19) in (26), we get (20) .

Then, the proof is complete.

\section{General Decay and Polynomial Decay}

In the previous work, it was supposed that $h^{\prime}(t) \leq 0$. Therefore, from $(20)$, we see that $e^{\prime}(t) \leq 0$. This implies that $e(t) \leq e(0)$, for all $t \geq 0$. In our case, we are not assuming that $h^{\prime}(t) \leq 0$. In fact, we are allowing the function $h(t)$ to oscillate.

To prove our result, we need to introduce the following auxiliary functional:

$$
\Phi(t):=\frac{1}{\rho+1} \int_{\Omega}\left|u_{t}\right|^{\rho} u_{t}(t) u(t) d x
$$

and:

$$
\begin{aligned}
\Psi(t): & =\int_{\Omega} \int_{0}^{t} L_{\alpha}(t-s)|\Delta u(t)-\Delta u(s)|^{2} d s d x \\
: & =\left(L_{\alpha} \square \Delta u\right)(t),
\end{aligned}
$$

where:

$$
\begin{aligned}
L_{\alpha}(t): & =e^{-\alpha t} \int_{t}^{+\infty} L_{\alpha}(s) d s \\
: & =e^{-\alpha t} \int_{t}^{+\infty} l(s) e^{\alpha s} d s
\end{aligned}
$$

and:

$$
\int_{0}^{\infty} L_{\alpha}^{1-\tau}(s) d s<\infty \text {, for } 0<\tau<2-r<1 \text { where } 1<r<\frac{3}{2} .
$$


$l(t)$ is defined in (8). Further, we consider the functional:

$$
\begin{aligned}
V(t): & =e(t)+\varepsilon \xi(t) \Phi(t)+\varepsilon \xi(t)\left(\Delta u_{t}(t), \Delta u(t)\right)_{L^{2}(\Omega)} \\
& +\eta \xi(t) \Psi(t)-\eta \xi(t)\left(\int_{0}^{t} L_{\alpha}(s) d s\right)\|\Delta u(t)\|_{L^{2}(\Omega)}^{2} \\
& +2 \eta \xi(t)\left\{\int_{\Omega} \Delta u(t) \int_{0}^{t} L_{\alpha}(t-s) \Delta u(s) d s d x\right\} \\
& -2 k_{1} \xi(0)\left(\int_{0}^{t}\left\|u_{s}(s)\right\|_{\rho+2}^{\rho+2} d s\right)-2 k_{2} \xi(0)\left(\int_{0}^{t}\|\Delta u(s)\|_{L^{2}(\Omega)}^{2} d s\right) \\
& -2 k_{3} \xi(0)\left(\int_{0}^{t}\left\|\Delta u_{s}(s)\right\|_{L^{2}(\Omega)}^{2} d s\right)-k_{4} \xi(0)\left(\int_{0}^{t}\|u(s)\|_{\gamma}^{\gamma} d s\right) \\
& -2 k_{5} \xi(0)\left(\int_{0}^{t}\left(L_{\alpha}^{r} \square \Delta u\right)(s) d s\right),
\end{aligned}
$$

where $\left(1 \leq r<\frac{3}{2}\right)$ and for some positive constants $\varepsilon, \eta, k_{1}, k_{2}, k_{3}, k_{4}$, and $k_{5}$ to be determined later.

Proposition 1. Let:

$$
\begin{aligned}
H(t): & =V(t)+2 k_{1} \xi(0)\left(\int_{0}^{t}\left\|u_{s}(s)\right\|_{\rho+2}^{\rho+2} d s\right)+2 k_{2} \xi(0)\left(\int_{0}^{t}\|\Delta u(s)\|_{L^{2}(\Omega)}^{2} d s\right) \\
& +2 k_{3} \xi(0)\left(\int_{0}^{t}\left\|\Delta u_{s}(s)\right\|_{L^{2}(\Omega)}^{2} d s\right)+k_{4} \xi(0)\left(\int_{0}^{t}\|u(s)\|_{\gamma}^{\gamma} d s\right) \\
& +2 k_{5} \xi(0)\left(\int_{0}^{t}\left(L_{\alpha}^{r} \square \Delta u\right)(s) d s\right) \\
: & =e(t)+\varepsilon \xi(t) \Phi(t)+\varepsilon \xi(t)\left(\Delta u_{t}(t), \Delta u(t)\right)_{L^{2}(\Omega)} \\
& +\eta \xi(t) \Psi(t)-\eta \xi(t)\left(\int_{0}^{t} L_{\alpha}(s) d s\right)\|\Delta u(t)\|_{L^{2}(\Omega)}^{2} \\
& +2 \eta \xi(t)\left\{\int_{\Omega} \Delta u(t) \int_{0}^{t} L_{\alpha}(t-s) \Delta u(s) d s d x\right\},
\end{aligned}
$$

then there exist positive constants $C_{1}$ and $C_{2}$ such that:

$$
C_{1}\{E(t)+(h \square \Delta u)(t)\} \leq H(t) \leq C_{2}\{E(t)+(h \square \Delta u)(t)+\Psi(t)\} .
$$

Proof. For the function $\Phi(t)$ definite in (27), by using Holder inequality (for $p=\frac{\rho+2}{\rho+1}$ and $q=\rho+2$ ), Young's inequality (for $\left.\varepsilon=\varepsilon_{1}\right),\left\|u_{t}(t)\right\|_{\rho+2}^{\rho} \leq[(\rho+2) e(0)]^{\frac{\rho}{(\rho+2)}}$, embedding $H^{1} \hookrightarrow L^{2(\rho+1)}$, and the Poincaré inequality, we get:

$$
\begin{aligned}
\Phi(t) & \leq \frac{1}{\rho+1}\left\|u_{t}(t)\right\|_{\rho+2}^{\rho+1}\|u(t)\|_{\rho+2} \\
& \leq \frac{\varepsilon_{1}}{2(\rho+1)^{2}}\left\|u_{t}(t)\right\|_{\rho+2}^{2(\rho+1)}+\frac{1}{2 \varepsilon_{1}}\|u(t)\|_{\rho+2}^{2} \\
& =\frac{\varepsilon_{1}}{2(\rho+1)^{2}}\left\|u_{t}(t)\right\|_{\rho+2}^{\rho}\left\|u_{t}(t)\right\|_{\rho+2}^{\rho+2}+\frac{1}{2 \varepsilon_{1}}\|u(t)\|_{\rho+2}^{2} \\
& \leq \frac{\varepsilon_{1}[(\rho+2) e(0)]}{2(\rho+1)^{2}}\left\|u_{t}(t)\right\|_{\rho+2}^{\rho+2}+\frac{C_{0} C_{p}}{2 \varepsilon_{1}}\|\Delta u(t)\|_{L^{2}(\Omega)}^{2},
\end{aligned}
$$

where $C_{0}$ comes from the embedding $H^{1} \hookrightarrow L^{2(\rho+1)}$ and the $C_{p}$ constant Poincaré inequality.

By multiplying (34) by $\varepsilon \xi(t)$ and using $0<\xi(t) \leq \xi(0)$, we get:

$$
\begin{aligned}
& \varepsilon \xi(t) \Phi(t) \\
\leq & \frac{\varepsilon \xi(0) \varepsilon_{1}[(\rho+2) e(0)]^{\frac{\rho}{(\rho+2)}}}{2(\rho+1)^{2}}\left\|u_{t}(t)\right\|_{\rho+2}^{\rho+2}+\frac{\varepsilon \xi(0) C_{0} C_{p}}{2 \varepsilon_{1}}\|\Delta u(t)\|_{L^{2}(\Omega)}^{2} .
\end{aligned}
$$

By using Young's inequality (for $\varepsilon=\varepsilon_{2}$ ) and using $0<\xi(t) \leq \xi(0)$, we get:

$$
\begin{aligned}
& \varepsilon \mathcal{\xi}(t)\left(\Delta u_{t}(t), \Delta u(t)\right)_{L^{2}(\Omega)} \\
\leq & \frac{\varepsilon \mathcal{\varepsilon}(0) \varepsilon_{2}}{2}\left\|\Delta u_{t}(t)\right\|_{L^{2}(\Omega)}^{2}+\frac{\varepsilon \mathcal{\xi}(0)}{2 \varepsilon_{2}}\|\Delta u(t)\|_{L^{2}(\Omega)}^{2} .
\end{aligned}
$$


Note that from (8) and (29), we get:

$$
\begin{aligned}
\int_{0}^{t} L_{\alpha}(s) d s & \leq \frac{1}{\alpha} \int_{0}^{\infty} l(s) e^{\alpha s} d s \\
& :=\frac{\bar{l}_{\alpha}}{\alpha}
\end{aligned}
$$

then, by multiplying (37) by $\xi(t)$ and using $0<\xi(t) \leq \xi(0)$, we get:

$$
-\eta \xi(t)\left(\int_{0}^{t} L_{\alpha}(s) d s\right)\|\Delta u(t)\|_{L^{2}(\Omega)}^{2} \leq \frac{\eta \xi(0) \bar{l}_{\alpha}}{\alpha}\|\Delta u(t)\|_{L^{2}(\Omega)}^{2} .
$$

By using Young's inequality (for $\varepsilon=\frac{\varepsilon_{3}}{2}$ ) and (28), we get:

$$
\begin{aligned}
& \int_{\Omega} \Delta u(t) \int_{0}^{t} L_{\alpha}(t-s) \Delta u(s) d s d x \\
= & \int_{0}^{t} L_{\alpha}(t-s)\left[\int_{\Omega} \Delta u(s) \Delta u(t) d x\right] d s \\
= & \int_{0}^{t} L_{\alpha}(t-s)\left[\int_{\Omega}[\Delta u(s)-\Delta u(t)+\Delta u(t)] \Delta u(t) d x\right] d s \\
= & \int_{0}^{t} L_{\alpha}(t-s)\left[\int_{\Omega}[\Delta u(s)-\Delta u(t)] \Delta u(t) d x\right] d s \\
& +\int_{0}^{t} L_{\alpha}(t-s)\left[\int_{\Omega}|\Delta u(t)|^{2} d x\right] d s \\
\leq \quad & \frac{1}{4 \varepsilon_{3}} \int_{0}^{t} L_{\alpha}(t-s)\left[\int_{\Omega}|\Delta u(s)-\Delta u(t)|^{2} d x\right] d s \\
& +\varepsilon_{3} \int_{0}^{t} L_{\alpha}(t-s)\left[\int_{\Omega}|\Delta u(t)|^{2} d x\right] d s \\
& +\left(\int_{0}^{t} L_{\alpha}(s) d s\right)\|\Delta u(t)\|_{L^{2}(\Omega)}^{2} \\
= & \frac{1}{4 \varepsilon_{3}} \Psi(t)+\left(1+\varepsilon_{3}\right)\left(\int_{0}^{t} L_{\alpha}(s) d s\right)\|\Delta u(t)\|_{L^{2}(\Omega)}^{2} .
\end{aligned}
$$

By multiplying (39) by $\xi(t)$ and using $0<\xi(t) \leq \xi(0)$ and (37), we get:

$$
\begin{aligned}
& 2 \eta \xi(t)\left\{\int_{\Omega} \Delta u(t) \int_{0}^{t} L_{\alpha}(t-s) \Delta u(s) d s d x\right\} \\
\leq \quad & \frac{\eta}{2 \varepsilon_{3}} \xi(t) \Psi(t)+\frac{2 \eta\left(1+\varepsilon_{3}\right) \xi(0) \bar{l}_{\alpha}}{\alpha}\|\Delta u(t)\|_{L^{2}(\Omega)}^{2} .
\end{aligned}
$$

By using (19) , (35), (36), (38) and (40) in (32), we get:

$$
\begin{aligned}
H(t) \geq & \left\{\frac{1}{\rho+2}-\frac{\varepsilon \xi(0) \varepsilon_{1}[(\rho+2) e(0)]^{\frac{\rho}{(\rho+2)}}}{2(\rho+1)^{2}}\right\}\left\|u_{t}(t)\right\|_{\rho+2}^{\rho+2} \\
& +\left\{\frac{\left(\xi_{0}-\bar{h}\right)}{2}-\frac{\varepsilon \xi(0) C_{0} C_{p}}{2 \varepsilon_{1}}-\frac{\varepsilon \xi(0)}{2 \varepsilon_{2}}\right. \\
& \left.-\frac{\eta \xi^{2}(0) \bar{l}_{\alpha}}{\alpha}-\frac{2 \eta\left(1+\varepsilon_{3}\right) \xi(0) \bar{l}_{\alpha}}{\alpha}\right\}\|\Delta u(t)\|_{L^{2}(\Omega)}^{2} \\
& +\frac{\xi_{1}}{2(\beta+1)}\|\Delta u(t)\|_{L^{2}(\Omega+1)}^{2(\beta)}+\left\{\frac{1}{2}-\frac{\varepsilon \xi(0) \varepsilon_{2}}{2}\right\}\left\|\Delta u_{t}(t)\right\|_{L^{2}(\Omega)}^{2} \\
& -\frac{1}{\gamma}\left(\int_{\Omega}|u(t)|^{\gamma} \ln |u|^{k} d x\right)+\frac{k}{\gamma^{2}}\|u(t)\|_{\gamma}^{\gamma}+\frac{1}{2}(h \square \Delta u)(t) \\
& +\eta\left(1-\frac{1}{2 \varepsilon_{3}}\right) \xi(t) \Psi(t) .
\end{aligned}
$$


Clearly, choosing:

$$
\left\{\begin{array}{l}
\varepsilon_{1}:=\frac{(\rho+1)^{2}}{(\rho+2) \varepsilon \xi(0)[(\rho+2) e(0)]^{\frac{\rho}{(\rho+2)}}}, \\
\varepsilon_{2}:=\frac{1}{2 \varepsilon \xi(0)}, \\
\varepsilon_{3}:=1, \\
\varepsilon<\frac{(\rho+1) \sqrt{\left(\xi_{0}-\bar{h}\right)}}{\xi(0) \sqrt{3 C_{0} C_{p}(\rho+2)[(\rho+2) e(0)]^{\frac{\rho}{(\rho+2)}}+6(\rho+1)^{2}}}, \\
\eta<\frac{\alpha\left(\xi_{0}-\bar{h}\right)}{30 \xi(0) \bar{l}_{\alpha}} .
\end{array}\right.
$$

By using (43) in (42), we get:

$$
\begin{aligned}
H(t) \geq & \frac{1}{2(\rho+2)}\left\|u_{t}(t)\right\|_{\rho+2}^{\rho+2}+\frac{\left(\xi_{0}-\bar{h}\right)}{6}\|\Delta u(t)\|_{L^{2}(\Omega)}^{2} \\
& +\frac{\xi_{1}}{2(\beta+1)}\|\Delta u(t)\|_{L^{2}(\Omega)}^{2(\beta+1)}+\frac{1}{4}\left\|\Delta u_{t}(t)\right\|_{L^{2}(\Omega)}^{2} \\
& -\frac{1}{\gamma}\left(\int_{\Omega}|u(t)|^{\gamma} \ln |u|^{k} d x\right)+\frac{k}{\gamma^{2}}\|u(t)\|_{\gamma}^{\gamma}+\frac{1}{2}(h \square \Delta u)(t) .
\end{aligned}
$$

Then, $\exists C_{1}>0$, where:

$$
H(t) \geq C_{1}\{E(t)+(h \square \Delta u)(t)\} .
$$

On the other hand, by replacing (19), (35), (36) , (38) and (40) in (32) and taking $\varepsilon_{1}=\varepsilon_{2}=$ $\varepsilon_{3}=1$, we get:

$$
\begin{aligned}
H(t) \leq & \left\{\frac{1}{\rho+2}+\frac{\varepsilon \xi(0)[(\rho+2) e(0)]^{\frac{\rho}{(\rho+2)}}}{2(\rho+1)^{2}}\right\}\left\|u_{t}(t)\right\|_{\rho+2}^{\rho+2} \\
& +\left\{\frac{\left(\xi_{0}+\varepsilon \xi(0) C_{0} C_{p}+\varepsilon \xi(0)\right)}{2}+\frac{4 \eta \xi(0) \bar{l}_{\alpha}}{\alpha}\right\}\|\Delta u(t)\|_{L^{2}(\Omega)}^{2} \\
& +\frac{\xi_{1}}{2(\beta+1)}\|\Delta u(t)\|_{L^{2}(\Omega)}^{2(\beta+1)} \\
& +\frac{\{1+\varepsilon \xi(0)\}}{2}\left\|\Delta u_{t}(t)\right\|_{L^{2}(\Omega)}^{2}-\frac{1}{\gamma}\left(\int_{\Omega}|u(t)|^{\gamma} \ln |u|^{k} d x\right) \\
& +\frac{k}{\gamma^{2}}\|u(t)\|_{\gamma}^{\gamma}+\frac{1}{2}(h \square \Delta u)(t)+\frac{3 \eta}{2} \xi(0) \Psi(t) .
\end{aligned}
$$

Then, $\exists C_{2}>0$, where:

$$
H(t) \leq C_{2}\{E(t)+(h \square \Delta u)(t)+\Psi(t)\},
$$

by using (43) and (44), we get (33).

The proof is complete. 
Lemma 3. For $r>1$ and $0<\theta<1$, we have:

$$
\begin{aligned}
& \int_{0}^{t} h(t-s)\|w(s)\|_{L^{2}(\Omega)}^{2} d s \\
\leq & \left(\int_{0}^{t} h^{1-\theta}(t-s)\|w(s)\|_{L^{2}(\Omega)}^{2} d s\right)^{\frac{1}{r}} \times\left(\int_{0}^{t} h^{\frac{(r-1+\theta)}{r-1}}(t-s)\|w(s)\|_{L^{2}(\Omega)}^{2} d s\right)^{\frac{r-1}{r}},
\end{aligned}
$$

for any $w \in L^{2}(\Omega)$.

Proof. It suffices to note that:

$$
\begin{aligned}
& \int_{0}^{t} h(t-s)\|w(s)\|_{L^{2}(\Omega)}^{2} d s \\
= & \int_{0}^{t} h^{\frac{(1-\theta)}{r}}(t-s)\|w(s)\|_{L^{2}(\Omega)}^{\frac{2}{r}} h^{\frac{(r-1+\theta)}{r}}(t-s)\|w(s)\|_{L^{2}(\Omega)}^{\frac{2(r-1)}{r}} d s,
\end{aligned}
$$

and applying Holder's inequality for

$$
p=r, q=\frac{r}{r-1}, r>1 \text {. }
$$

The proof of the lemma is completed.

Lemma 4. Let $v \in L^{\infty}\left((0, T) ; L^{2}(\Omega)\right)$ be such that $v_{x} \in L^{\infty}\left((0, t) ; L^{2}(\Omega)\right)$ and $h$ be a continuous function on $[0, T]$ and suppose that $0<\theta<1$ and $\rho>1$. Then, there exists a constant $C>0$ such that:

$$
\begin{aligned}
& \int_{0}^{t} h(t-s)\left\|v_{x}(., t)-v_{x}(., s)\right\|_{L^{2}(\Omega)}^{2} d s \\
\leq & C\left(\sup _{0<s<T}\|v(., s)\|_{L^{2}(\Omega)}^{2} \int_{0}^{t} h^{1-\theta}(s) d s\right)^{\frac{\rho-1}{\rho-1+\theta}} \\
& \times\left(\int_{0}^{t} h^{\rho}(t-s)\left\|v_{x}(., t)-v_{x}(., s)\right\|_{L^{2}(\Omega)}^{2} d s\right)^{\frac{\theta}{\rho-1+\theta}} .
\end{aligned}
$$

Proof. By using Lemma 3 with $r:=\frac{(\rho-1+\theta)}{(\rho-1)}$, we obtain:

$$
\begin{aligned}
& \int_{0}^{t} h(t-s)\left\|v_{x}(., t)-v_{x}(., s)\right\|_{L^{2}(\Omega)}^{2} d s \\
\leq & \left(\int_{0}^{t} h^{1-\theta}(t-s)\left\|v_{x}(., t)-v_{x}(., s)\right\|_{L^{2}(\Omega)}^{2} d s\right)_{\theta}^{\frac{\rho-1}{\rho-1+\theta}} \\
& \times\left(\int_{0}^{t} h^{\rho}(t-s)\left\|v_{x}(., t)-v_{x}(., s)\right\|_{L^{2}(\Omega)}^{2}\right)^{\frac{\theta_{\theta}}{\rho-1+\theta}} .
\end{aligned}
$$

It is easy to see that:

$$
\begin{aligned}
& \int_{0}^{t} h^{1-\theta}(t-s)\left\|v_{x}(., t)-v_{x}(., s)\right\|_{L^{2}(\Omega)}^{2} d s \\
\leq & C \sup _{0<s<T}\left\|v_{x}(., s)\right\|_{L^{2}(\Omega)}^{2} \int_{0}^{t} h^{1-\theta}(s) d s .
\end{aligned}
$$

By combining (45) and (46), the proof of the lemma is completed. 
Lemma 5. Let $v \in L^{\infty}\left((0, T) ; L^{2}(\Omega)\right)$ be such that $v_{x} \in L^{\infty}\left((0, T) ; L^{2}(\Omega)\right)$ and $h$ be a continuous function on $[0, T]$ and suppose that $\rho>1$. Then, there exists a constant $C>0$ such that:

$$
\begin{aligned}
& \int_{0}^{t} h(t-s)\left\|v_{x}(., t)-v_{x}(., s)\right\|_{L^{2}(\Omega)}^{2} d s \\
\leq & c\left(t\left\|v_{x}(., t)\right\|_{H}^{2}+\int_{0}^{t}\left\|v_{x}(., s)\right\|_{L^{2}(\Omega)}^{2} d s\right)^{\frac{\rho-1}{\rho}} \\
& \times\left(\int_{0}^{t} h^{\rho}(t-s)\left\|v_{x}(., t)-v_{x}(., s)\right\|_{L^{2}(\Omega)}^{2} d s\right)^{\frac{1}{\rho}} .
\end{aligned}
$$

Proof. We use (45) for $\theta=1$ to arrive at:

$$
\begin{aligned}
& \int_{0}^{t} h(t-s)\left\|v_{x}(., t)-v_{x}(., s)\right\|_{L^{2}(\Omega)}^{2} d s \\
\leq & \left(\int_{0}^{t}\left\|v_{x}(., t)-v_{x}(., s)\right\|_{L^{2}(\Omega)}^{2} d s\right)^{\frac{\rho-1}{\rho}} \\
& \times\left(\int_{0}^{t} h^{\rho}(t-s)\left\|v_{x}(., t)-v_{x}(., s)\right\|_{L^{2}(\Omega)}^{2} d s\right)^{\frac{1}{\rho}} .
\end{aligned}
$$

It suffices to note that:

$$
\begin{aligned}
& \int_{0}^{t}\left\|v_{x}(., t)-v_{x}(., s)\right\|_{L^{2}(\Omega)}^{2} d s \\
\leq \quad & 2 t\left\|v_{x}(., t)\right\|_{L^{2}(\Omega)}^{2}+2 \int_{0}^{t}\left\|v_{x}(., s)\right\|_{L^{2}(\Omega)}^{2} d s,
\end{aligned}
$$

to obtain (47).

This completes the proof.

Let:

$$
\varepsilon:=\min \left\{\frac{(\rho+1) \sqrt{\left(\xi_{0}-\bar{h}\right)}}{\xi(0) \sqrt{3 C_{0} C_{p}(\rho+2)[(\rho+2) e(0)]^{\frac{\rho}{(\rho+2)}}+6(\rho+1)^{2}}}, \frac{\left(\xi_{0}-\bar{h}\right)}{4\left(\int_{0}^{t} h^{2-r}(s) d s\right)}\right\}
$$

and:

$$
\bar{l}_{\alpha}<\frac{\alpha\left(\xi_{0}-\bar{h}\right)}{12 \alpha+6 L} \varepsilon
$$

Now, we are in a position to state and prove our first result.

Theorem 1. Assume that the hypotheses (A1)-(A3) hold, the initial data $\left(u_{0}, u_{1}\right)$ satisfy $E(0)>0$, and $\bar{l}_{\alpha}$ is as above.

Then, the classical energy $E(t)$ of (1)-(3) decays to zero exponentially and polynomially. That is, there exist positive constants $C_{1}, C_{2}$, and $C_{3}$ such that:

$$
E(t) \leq\left\{\begin{array}{l}
\frac{V(0)}{C_{1}} e^{-\frac{C_{3}}{C_{2}}\left(\int_{0}^{t} \xi(s) d s\right)}, \quad r=1, \quad t \geq 0 \\
C\left(1+\int_{0}^{t} \xi(s) d s\right)^{\frac{-1}{r-1}}, \quad 1<r<\frac{3}{2}, \quad t \geq 0 .
\end{array}\right.
$$


Proof. Now, a differentiation of $V(t)$ definite in (31) with respect to time gives:

$$
\begin{aligned}
& \frac{d}{d t}\left\{V(t)+k_{1} \xi(0)\left(\int_{0}^{t}\left\|u_{s}(s)\right\|_{\rho+2}^{\rho+2} d s\right)+k_{2} \xi(0)\left(\int_{0}^{t}\|\Delta u(s)\|_{L^{2}(\Omega)}^{2} d s\right)\right. \\
& +k_{3} \xi(0)\left(\int_{0}^{t}\left\|\Delta u_{s}(s)\right\|_{L^{2}(\Omega)}^{2} d s\right)+k_{4} \xi(0)\left(\int_{0}^{t}\|u(s)\|_{\gamma}^{\gamma} d s\right) \\
& \left.+k_{5} \xi(0)\left(\int_{0}^{t}\left(L_{\alpha}^{r} \square \Delta u\right)(s) d s\right)\right\} \\
= & \frac{d}{d t}\{e(t)\}+\varepsilon \frac{d}{d t}\left\{\xi(t) \Phi(t)+\xi(t)\left(\Delta u_{t}(t), \Delta u(t)\right)_{L^{2}(\Omega)}\right\} \\
& +\eta \frac{d}{d t}\left\{\xi(t) \psi(t)-\xi(t)\left(\int_{0}^{t} L_{\alpha}(s) d s\right)\|\Delta u(t)\|_{L^{2}(\Omega)}^{2}\right. \\
& \left.+2 \xi(t) \int_{\Omega} \Delta u(t) \int_{0}^{t} L_{\alpha}(t-s) \Delta u(s) d s d x\right\} \\
& -k_{1} \xi(0)\left\|u_{t}(t)\right\|_{\rho+2}^{\rho+2}-k_{2} \xi(0)\|\Delta u(t)\|_{L^{2}(\Omega)}^{2}-k_{3} \xi(0)\left\|\Delta u_{t}(t)\right\|_{L^{2}(\Omega)}^{2} \\
& -k_{4} \xi(0)\|u(t)\|_{\gamma}^{\gamma}-k_{5} \xi(0)\left(L_{\alpha}^{r} \square \Delta u\right)(t) .
\end{aligned}
$$

By a differentiation of (27), we have:

$$
\begin{aligned}
& \frac{d}{d t}\{\xi(t) \Phi(t)\} \\
= & \frac{1}{\rho+1} \xi^{\prime}(t)\left(\int_{\Omega}\left|u_{t}\right|^{\rho} u_{t}(t) u(t) d x\right)+\frac{1}{\rho+1} \xi(t)\left(\int_{\Omega}\left|u_{t}\right|^{\rho} u_{t}(t) u_{t}(t) d x\right) \\
& +\frac{1}{\rho+1} \xi(t)\left(\int_{\Omega} \frac{d}{d t}\left\{\left|u_{t}\right|^{\rho}\right\} u_{t}(t) u(t) d x\right) \\
& +\frac{1}{\rho+1} \xi(t)\left(\int_{\Omega}\left|u_{t}\right|^{\rho} u_{t t}(t) u(t) d x\right) \\
= & \frac{1}{\rho+1} \xi^{\prime}(t)\left(\int_{\Omega}\left|u_{t}\right|^{\rho} u_{t}(t) u(t) d x\right)+\frac{1}{\rho+1} \xi(t)\left\|u_{t}(t)\right\|_{\rho+2}^{\rho+2} \\
& +\frac{\rho}{\rho+1} \xi(t)\left(\int_{\Omega}\left|u_{t}\right|^{\rho-2} u_{t t}(t) u_{t}(t) u_{t}(t) u(t) d x\right) \\
& +\frac{1}{\rho+1} \xi(t)\left(\int_{\Omega}\left|u_{t}\right|^{\rho} u_{t t}(t) u(t) d x\right) \\
= & \frac{1}{\rho+1} \xi^{\prime}(t)\left(\int_{\Omega}\left|u_{t}\right|^{\rho} u_{t}(t) u(t) d x\right)+\frac{1}{\rho+1} \xi(t)\left\|u_{t}(t)\right\|_{\rho+2}^{\rho+2} \\
& +\xi(t)\left(\int_{\Omega}\left|u_{t}\right|^{\rho} u_{t t}(t) u(t) d x\right) .
\end{aligned}
$$

By using $u$ as a solution in (1)-(3), we get:

$$
\begin{aligned}
& \xi(t)\left(\int_{\Omega}\left|u_{t}\right|^{\rho} u_{t t}(t) u(t) d x\right) \\
= & -\xi(t) \int_{\Omega}\left(\xi_{0}+\xi_{1}\|\Delta u(t)\|_{L^{2}(\Omega)}^{2 \beta}\right) \Delta^{2} u(t) u(t) d x \\
& -\xi(t)\left(\int_{\Omega} \Delta^{2} u_{t t}(t) u(t) d x\right) \\
& +\xi(t) \int_{\Omega} u(t)\left[\int_{0}^{t} h(t-s) \Delta^{2} u(s) d s\right] d x \\
& +\xi(t)\left(\int_{\Omega} u|u|^{\gamma-2} \ln |u|^{k} u(t) d x\right) .
\end{aligned}
$$

Integrating by parts, we get:

$$
\begin{aligned}
& -\xi(t) \int_{\Omega}\left(\xi_{0}+\xi_{1}\|\Delta u(t)\|_{L^{2}(\Omega)}^{2 \beta}\right) \Delta^{2} u(t) u(t) d x \\
= & -\xi(t)\left(\xi_{0}+\xi_{1}\|\Delta u(t)\|_{L^{2}(\Omega)}^{2 \beta}\right) \int_{\Omega} \Delta^{2} u(t) u(t) d x \\
= & -\xi(t)\left(\xi_{0}+\xi_{1}\|\Delta u(t)\|_{L^{2}(\Omega)}^{2 \beta}\right)\|\Delta u(t)\|_{L^{2}(\Omega)}^{2} \\
= & -\xi_{0} \xi(t)\|\Delta u(t)\|_{L^{2}(\Omega)}^{2}-\xi_{1} \xi(t)\|\Delta u(t)\|_{L^{2}(\Omega)}^{2(3+1)} .
\end{aligned}
$$

Also integrating by parts, we get:

$$
\begin{aligned}
& -\xi(t)\left(\int_{\Omega} \Delta^{2} u_{t t}(t) u(t) d x\right) \\
= & -\xi(t)\left(\int_{\Omega} \Delta u_{t t}(t) \Delta u(t) d x\right) \\
= & -\xi(t) \frac{d}{d t}\left\{\left(\Delta u_{t}(t), \Delta u(t)\right)_{L^{2}(\Omega)}\right\}+\xi(t)\left\|\Delta u_{t}(t)\right\|_{L^{2}(\Omega)}^{2} \\
= & -\frac{d}{d t}\left\{\xi(t)\left(\Delta u_{t}(t), \Delta u(t)\right)_{L^{2}(\Omega)}\right\}+\xi^{\prime}(t)\left(\Delta u_{t}(t), \Delta u(t)\right)_{L^{2}(\Omega)} \\
& +\xi(t)\left\|\Delta u_{t}(t)\right\|_{L^{2}(\Omega)}^{2} .
\end{aligned}
$$


By using integration by parts, we get:

$$
\begin{aligned}
& \xi(t) \int_{\Omega} u(t)\left[\int_{0}^{t} h(t-s) \Delta^{2} u(s) d s\right] d x \\
= & \xi(t)\left\{\int_{\Omega} \Delta u(t) \int_{0}^{t} h(t-s) \Delta u(s) d s d x\right\} .
\end{aligned}
$$

By direct calculation, we get:

$$
\xi(t)\left(\int_{\Omega} u|u|^{\gamma-2} \ln |u|^{k} u(t) d x\right)=\xi(t)\left(\int_{\Omega}|u(t)|^{\gamma} \ln |u|^{k} d x\right) .
$$

By replacing (53)-(56) in (52), we get:

$$
\begin{aligned}
& \xi(t)\left(\int_{\Omega}\left|u_{t}\right|^{\rho} u_{t t}(t) u(t) d x\right) \\
= & -\xi_{0} \xi(t)\|\Delta u(t)\|_{L^{2}(\Omega)}^{2}-\xi 1 \xi(t)\|\Delta u(t)\|_{L^{2}(\Omega)}^{2(\beta+1)} \\
& -\frac{d}{d t}\left\{\xi(t)\left(\Delta u_{t}(t), \Delta u(t)\right)_{L^{2}(\Omega)}\right\} \\
& +\xi^{\prime}(t)\left(\Delta u_{t}(t), \Delta u(t)\right)_{L^{2}(\Omega)}+\xi(t)\left\|\Delta u_{t}(t)\right\|_{L^{2}(\Omega)}^{2} \\
& +\xi(t)\left\{\int_{\Omega} \Delta u(t) \int_{0}^{t} h(t-s) \Delta u(s) d s d x\right\} \\
& +\xi(t)\left\{\int_{\Omega}|u(t)|^{\gamma} \ln |u|^{k} d x\right) .
\end{aligned}
$$

By replacing (57) in (51), we get:

$$
\begin{aligned}
& \frac{d}{d t}\{\xi(t) \Phi(t)\} \\
= & \frac{1}{\rho+1} \xi^{\prime}(t)\left(\int_{\Omega}\left|u_{t}\right|^{\rho} u_{t}(t) u(t) d x\right)+\frac{1}{\rho+1} \xi(t)\left\|u_{t}(t)\right\|_{\rho+2}^{\rho+2} \\
& -\xi_{0} \xi(t)\|\Delta u(t)\|_{L^{2}(\Omega)}^{2}-\xi_{1} \xi(t)\|\Delta u(t)\|_{L^{2}(\Omega)}^{2(\beta+1)} \\
& -\frac{d}{d t}\left\{\xi(t)\left(\Delta u_{t}(t), \Delta u(t)\right)_{L^{2}(\Omega)}\right\} \\
& +\xi^{\prime}(t)\left(\Delta u_{t}(t), \Delta u(t)\right)_{L^{2}(\Omega)}+\xi(t)\left\|\Delta u_{t}(t)\right\|_{L^{2}(\Omega)}^{2} \\
& +\xi(t)\left\{\int_{\Omega} \Delta u(t) \int_{0}^{t} h(t-s) \Delta u(s) d s d x\right\}+\xi(t)\left(\int_{\Omega}|u(t)|^{\gamma} \ln |u|^{k} d x\right),
\end{aligned}
$$

then (58) is equivalent to:

$$
\begin{aligned}
& \frac{d}{d t}\left\{\xi(t) \Phi(t)+\xi(t)\left(\Delta u_{t}(t), \Delta u(t)\right)_{L^{2}(\Omega)}\right\} \\
= & \frac{1}{\rho+1} \xi^{\prime}(t)\left(\int_{\Omega}\left|u_{t}\right|^{\rho} u_{t}(t) u(t) d x\right)+\frac{1}{\rho+1} \xi(t)\left\|u_{t}(t)\right\|_{\rho+2}^{\rho+2} \\
& -\xi_{0} \xi^{\prime}(t)\|\Delta u(t)\|_{L^{2}(\Omega)}^{2}-\xi 1 \xi(t)\|\Delta u(t)\|_{L^{2}(\Omega)}^{2(\beta+1)} \\
& +\xi^{\prime}(t)\left(\Delta u_{t}(t), \Delta u(t)\right)_{L^{2}(\Omega)}+\xi(t)\left\|\Delta u_{t}(t)\right\|_{L^{2}(\Omega)}^{2} \\
& +\xi(t)\left\{\int_{\Omega} \Delta u(t) \int_{0}^{t} h(t-s) \Delta u(s) d s d x\right\}+\xi(t)\left(\int_{\Omega}|u|^{\gamma} \ln |u|^{k} d x\right)
\end{aligned}
$$

and:

$$
\frac{d}{d t}\{\xi(t) \Psi(t)\}=\xi^{\prime}(t) \Psi(t)+\xi(t) \frac{d}{d t}\{\Psi(t)\} .
$$

On the other hand, we have:

$$
\begin{aligned}
\frac{d}{d t}\{\Psi(t)\}= & \int_{\Omega} \int_{0}^{t} \frac{d}{d t}\left\{L_{\alpha}(t-s)\right\}|\Delta u(t)-\Delta u(s)|^{2} d s d x \\
& +\int_{\Omega} \int_{0}^{t} L_{\alpha}(t-s) \frac{d}{d t}\left\{|\Delta u(t)-\Delta u(s)|^{2}\right\} d s d x .
\end{aligned}
$$


By using:

$$
\begin{aligned}
\frac{d}{d t}\left\{L_{\alpha}(t-s)\right\} & =\frac{d}{d t}\left\{e^{-\alpha(t-s)} \int_{(t-s)}^{+\infty} l(y) e^{\alpha y} d y\right\} \\
& =-\alpha e^{-\alpha(t-s)} \int_{(t-s)}^{+\infty} l(y) e^{\alpha y} d y-e^{-\alpha(t-s)} l(t-s) e^{\alpha(t-s)} \\
& =-\alpha L_{\alpha}(t-s)-l(t-s)
\end{aligned}
$$

we get:

$$
\begin{aligned}
& \int_{\Omega} \int_{0}^{t} \frac{d}{d t}\left\{L_{\alpha}(t-s)\right\}|\Delta u(t)-\Delta u(s)|^{2} d s d x \\
= & \int_{\Omega} \int_{0}^{t}\left\{-\alpha L_{\alpha}(t-s)-l(t-s)\right\}|\Delta u(t)-\Delta u(s)|^{2} d s d x \\
= & -\alpha \int_{\Omega} \int_{0}^{t} L_{\alpha}(t-s)|\Delta u(t)-\Delta u(s)|^{2} d s d x \\
& -\int_{\Omega} \int_{0}^{t} l(t-s)|\Delta u(t)-\Delta u(s)|^{2} d s d x \\
= & -\alpha \Psi(t)-(l \square \Delta u)(t) .
\end{aligned}
$$

By direct calculation, we get:

$$
\begin{aligned}
& \int_{\Omega} \int_{0}^{t} L_{\alpha}(t-s) \frac{d}{d t}\left\{|\Delta u(t)-\Delta u(s)|^{2}\right\} d s d x \\
= & 2 \int_{\Omega} \int_{0}^{t} L_{\alpha}(t-s) \Delta u_{t}(t)(\Delta u(t)-\Delta u(s)) d s d x \\
= & 2\left(\int_{0}^{t} L_{\alpha}(s) d s\right) \int_{\Omega} \Delta u(t) . \Delta u_{t}(t) d x \\
& -2\left\{\int_{\Omega} \Delta u_{t}(t) \int_{0}^{t} L_{\alpha}(t-s) \Delta u(s) d s d x\right\} .
\end{aligned}
$$

Replacing (62) and (63) in (61), we get:

$$
\begin{aligned}
\frac{d}{d t}\{\Psi(t)\}= & -\alpha \Psi(t)-(l \square \Delta u)(t)+2\left(\int_{0}^{t} L_{\alpha}(s) d s\right) \int_{\Omega} \Delta u(t) . \Delta u_{t}(t) d x \\
& -2\left\{\int_{\Omega} \Delta u_{t}(t) \int_{0}^{t} L_{\alpha}(t-s) \Delta u(s) d s d x\right\} .
\end{aligned}
$$

By replacing (64) in (60), we get:

$$
\begin{aligned}
& \frac{d}{d t}\{\xi(t) \Psi(t)\} \\
= & \xi^{\prime}(t) \Psi(t)-\alpha \xi(t) \Psi(t)-\xi(t)(l \square \Delta u)(t) \\
& +2\left(\int_{0}^{t} L_{\alpha}(s) d s\right) \xi(t) \int_{\Omega} \Delta u(t) \cdot \Delta u_{t}(t) d x \\
& -2 \xi(t)\left\{\int_{\Omega} \Delta u_{t}(t) \int_{0}^{t} L_{\alpha}(t-s) \Delta u(s) d s d x\right\} .
\end{aligned}
$$

By using direct calculation, we get:

$$
\begin{aligned}
& 2\left(\int_{0}^{t} L_{\alpha}(s) d s\right) \xi(t) \int_{\Omega} \Delta u(t) \cdot \Delta u_{t}(t) d x \\
= & \left(\int_{0}^{t} L_{\alpha}(s) d s\right) \xi(t) \frac{d}{d t}\left\{\|\Delta u(t)\|_{L^{2}(\Omega)}^{2}\right\} \\
= & \frac{d}{d t}\left\{\left(\int_{0}^{t} L_{\alpha}(s) d s\right) \xi(t)\|\Delta u(t)\|_{L^{2}(\Omega)}^{2}\right\} \\
& -\frac{d}{d t}\left\{\left(\int_{0}^{t} L_{\alpha}(s) d s\right) \xi(t)\right\}\|\Delta u(t)\|_{L^{2}(\Omega)}^{2} \\
= & \frac{d}{d t}\left\{\left(\int_{0}^{t} L_{\alpha}(s) d s\right) \xi(t)\|\Delta u(t)\|_{L^{2}(\Omega)}^{2}\right\} \\
& -\left\{L_{\alpha}(t) \xi(t)+\left(\int_{0}^{t} L_{\alpha}(s) d s\right) \xi^{\prime}(t)\right\}\|\Delta u(t)\|_{L^{2}(\Omega)}^{2} .
\end{aligned}
$$


By using direct calculation and $\frac{d}{d t}\left\{L_{\alpha}(t)\right\}=-\alpha L_{\alpha}(t)-l(t)$, we get:

$$
\begin{aligned}
& -2 \xi(t)\left\{\int_{\Omega} \Delta u_{t}(t) \int_{0}^{t} L_{\alpha}(t-s) \Delta u(s) d s d x\right\} \\
= & -2 \xi(t)\left\{\int_{\Omega} \frac{d}{d t}\{\Delta u(t)\} \int_{0}^{t} L_{\alpha}(t-s) \Delta u(s) d s d x\right\} \\
= & -2 \frac{d}{d t}\left\{\xi(t)\left(\int_{\Omega} \Delta u(t) \int_{0}^{t} L_{\alpha}(t-s) \Delta u(s) d s d x\right)\right\} \\
& +2 \xi^{\prime}(t)\left\{\int_{\Omega} \Delta u(t) \int_{0}^{t} L_{\alpha}(t-s) \Delta u(s) d s d x\right\} \\
& -2 \alpha \xi(t)\left\{\int_{\Omega} \Delta u(t) \int_{0}^{t} L_{\alpha}(t-s) \Delta u(s) d s d x\right\} \\
& -2 \xi(t)\left\{\int_{\Omega} \Delta u(t) \int_{0}^{t} l(t-s) \Delta u(s) d s d x\right\} \\
& +2 L_{\alpha}(0) \xi(t)\|\Delta u(t)\|_{L^{2}(\Omega)}^{2} .
\end{aligned}
$$

Using (66) and (67) in (65), we see that:

$$
\begin{aligned}
& \frac{d}{d t}\left\{\xi(t) \Psi(t)-\left(\int_{0}^{t} L_{\alpha}(s) d s\right) \xi(t)\|\Delta u(t)\|_{L^{2}(\Omega)}^{2}\right. \\
& \left.+2 \xi(t)\left(\int_{\Omega} \Delta u(t) \int_{0}^{t} L_{\alpha}(t-s) \Delta u(s) d s d x\right)\right\} \\
= & \left\{\xi^{\prime}(t)-\alpha \xi(t)\right\} \Psi(t)-\xi(t)(l \square \Delta u)(t) \\
& -\left\{L_{\alpha}(t) \xi(t)+\left(\int_{0}^{t} L_{\alpha}(s) d s\right) \xi^{\prime}(t)-2 L_{\alpha}(0) \xi(t)\right\}\|\Delta u(t)\|_{L^{2}(\Omega)}^{2} \\
& +2\left(\xi^{\prime}(t)-\alpha \xi(t)\right)\left\{\int_{\Omega} \Delta u(t) \int_{0}^{t} L_{\alpha}(t-s) \Delta u(s) d s d x\right\} \\
& -2 \xi(t)\left\{\int_{\Omega} \Delta u(t) \int_{0}^{t} l(t-s) \Delta u(s) d s d x\right\} .
\end{aligned}
$$

Taking into account (20) , (59), and (68) in (50), we obtain:

$$
\begin{aligned}
& \frac{d}{d t}\left\{V(t)+k_{1} \xi(0)\left(\int_{0}^{t}\left\|u_{s}(s)\right\|_{\rho+2}^{\rho+2} d s\right)+k_{2} \xi(0)\left(\int_{0}^{t}\|\Delta u(s)\|_{L^{2}(\Omega)}^{2} d s\right)\right. \\
& +k_{3} \xi(0)\left(\int_{0}^{t}\left\|\Delta u_{s}(s)\right\|_{L^{2}(\Omega)}^{2} d s\right)+k_{4} \xi(0)\left(\int_{0}^{t}\|u(s)\|_{\gamma}^{\gamma} d s\right) \\
& \left.+k_{5} \xi(0)\left(\int_{0}^{t}\left(L_{\alpha}^{r} \square \Delta u\right)(s) d s\right)\right\} \\
= & \frac{1}{2}\left(h^{\prime} \square \Delta u\right)(t)-\frac{1}{2} h(t)\|\Delta u(t)\|_{L^{2}(\Omega)}^{2}+\frac{\varepsilon}{\rho+1} \xi^{\prime}(t)\left(\int_{\Omega}\left|u_{t}\right|^{\rho} u_{t}(t) u(t) d x\right) \\
& +\frac{\varepsilon}{\rho+1} \xi(t)\left\|u_{t}(t)\right\|_{\rho+2}^{\rho+2}-\varepsilon \xi_{0} \xi(t)\|\Delta u(t)\|_{L^{2}(\Omega)}^{2}-\varepsilon \xi \xi \xi(t)\|\Delta u(t)\|_{L^{2}(\Omega)}^{2(\beta+1)} \\
& +\varepsilon \xi^{\prime}(t)\left(\Delta u_{t}(t), \Delta u(t)\right)_{L^{2}(\Omega)}+\varepsilon \xi(t)\left\|\Delta u_{t}(t)\right\|_{L^{2}(\Omega)}^{2} \\
& +\varepsilon \xi(t)\left\{\int_{\Omega} \Delta u(t) \int_{0}^{t} h(t-s) \Delta u(s) d s d x\right\}+\varepsilon \xi(t)\left(\int_{\Omega}|u(t)|^{\gamma} \ln |u|^{k} d x\right) \\
& +\eta\left\{\xi^{\prime}(t)-\alpha \xi(t)\right\} \Psi(t)-\eta \xi(t)(l \square \Delta u)(t) \\
& -\eta\left\{L_{\alpha}(t) \xi(t)+\left(\int_{0}^{t} L_{\alpha}(s) d s\right) \xi^{\prime}(t)-2 L_{\alpha}(0) \xi(t)\right\}\|\Delta u(t)\|_{L^{2}(\Omega)}^{2} \\
& +2 \eta\left(\xi^{\prime}(t)-\alpha \xi(t)\right)\left\{\int_{\Omega} \Delta u(t) \int_{0}^{t} L_{\alpha}(t-s) \Delta u(s) d s d x\right\} \\
& -2 \eta \xi(t)\left\{\int_{\Omega} \Delta u(t) \int_{0}^{t} l(t-s) \Delta u(s) d s d x\right\} \\
& -k_{1} \xi(0)\left\|u_{t}(t)\right\|_{\rho+2}^{\rho+2}-k_{2} \xi(0)\|\Delta u(t)\|_{L^{2}(\Omega)}^{2}-k_{3} \xi(0)\left\|\Delta u_{t}(t)\right\|_{L^{2}(\Omega)}^{2} \\
& -k_{4} \xi(0)\|u(t)\|_{\gamma}^{\gamma}-k_{5} \xi(0)\left(L_{\alpha}^{r} \square \Delta u\right)(t) .
\end{aligned}
$$

Next, we use the estimate (69).

By multiplying (34) by $\varepsilon \xi^{\prime}(t)$ and $\left|\frac{\xi^{\prime}(t)}{\zeta(t)}\right| \leq L$, we get:

$$
\begin{aligned}
& \frac{\varepsilon}{\rho+1} \xi^{\prime}(t)\left(\int_{\Omega}\left|u_{t}\right|^{\rho} u_{t}(t) u(t) d x\right) \\
\leq & \frac{\varepsilon L \delta_{1}[(\rho+2) e(0)] \frac{\rho}{(\rho+2)}}{2(\rho+1)^{2}} \xi(t)\left\|u_{t}(t)\right\|_{\rho+2}^{\rho+2}+\frac{\varepsilon L C_{0} C_{p}}{2 \delta_{1}} \xi(t)\|\Delta u(t)\|_{L^{2}(\Omega)}^{2} .
\end{aligned}
$$


By using Young's inequality (for $\varepsilon=\delta_{2}$ ) and $\left|\frac{\xi^{\prime}(t)}{\xi(t)}\right| \leq L$, we get:

$$
\begin{aligned}
& \varepsilon \xi^{\prime}(t)\left(\Delta u_{t}(t), \Delta u(t)\right)_{L^{2}(\Omega)} \\
\leq \quad & \frac{\varepsilon L \delta_{2}}{2} \xi(t)\left\|\Delta u_{t}(t)\right\|_{L^{2}(\Omega)}^{2}+\frac{\varepsilon L}{2 \delta_{2}} \xi(t)\|\Delta u(t)\|_{L^{2}(\Omega)}^{2} .
\end{aligned}
$$

By using Young's inequality (for $\left.\varepsilon=\eta_{1}\right)$ and $\left(\int_{0}^{t} h(s) d s\right) \leq \bar{h}$, we get:

$$
\begin{aligned}
& \xi(t) \int_{\Omega} \Delta u(t)\left(\int_{0}^{t} h(t-s) \Delta u(s) d s\right) d x \\
\leq & \frac{1}{4} \xi(t)\|\Delta u(t)\|_{L^{2}(\Omega)}^{2} \\
& +\xi(t) \int_{\Omega}\left(\int_{0}^{t} h(t-s)(|\Delta u(s)-\Delta u(t)|+|\Delta u(t)|) d s\right)^{2} d x \\
\leq & \frac{1}{4} \xi(t)\|\Delta u(t)\|_{L^{2}(\Omega)}^{2}+\left(1+\eta_{1}\right) \bar{h}^{2} \xi(t)\|\Delta u(t)\|_{L^{2}(\Omega)}^{2} \\
& +\left(1+\frac{1}{\eta_{1}}\right) \xi(t)\left(\int_{0}^{t} h^{2-r}(s) d s\right)\left(h^{r} \square \Delta u\right)(t) \\
= & \left(\frac{1}{4}+\left(1+\eta_{1}\right) \bar{h}^{2}\right) \xi(t)\|\Delta u(t)\|_{L^{2}(\Omega)}^{2} \\
& +\left(1+\frac{1}{\eta_{1}}\right) \xi(t)\left(\int_{0}^{t} h^{2-r}(s) d s\right)\left(h^{r} \square \Delta u\right)(t) .
\end{aligned}
$$

Choosing $\eta_{1}:=\frac{\left(\xi_{0}-\bar{h}\right)}{\bar{h}}$, hence $\left(1+\eta_{1}\right) \bar{h}^{2}=\bar{h}$ and $\left(1+\frac{1}{\eta_{1}}\right)=\frac{1}{\left(\xi_{0}-\bar{h}\right)}$, we get:

$$
\begin{aligned}
& \varepsilon \xi(t) \int_{\Omega} \Delta u(t)\left(\int_{0}^{t} h(t-s) \Delta u(s) d s\right) d x \\
\leq & \varepsilon\left(\frac{1}{4}+\bar{h}\right) \xi(t)\|\Delta u(t)\|_{L^{2}(\Omega)}^{2} \\
& +\frac{\varepsilon}{\left(\xi_{0}-\bar{h}\right)} \xi(t)\left(\int_{0}^{t} h^{2-r}(s) d s\right)\left(h^{r} \square \Delta u\right)(t) .
\end{aligned}
$$

By using Young's inequality (for $\varepsilon=1)$ and $\left(\int_{0}^{t} L_{\alpha}(s) d s\right) \leq \frac{\bar{l}_{\alpha}}{\alpha}$, we get:

$$
\begin{aligned}
& \int_{\Omega} \Delta u(t)\left(\int_{0}^{t} L_{\alpha}(t-s) \Delta u(s) d s\right) d x \\
\leq & \frac{1}{4}\|\Delta u(t)\|_{L^{2}(\Omega)}^{2} \\
& +\int_{\Omega}\left(\int_{0}^{t} L_{\alpha}(t-s)(|\Delta u(s)-\Delta u(t)|+|\Delta u(t)|) d s\right)^{2} d x \\
\leq & \frac{1}{4}\|\Delta u(t)\|_{L^{2}(\Omega)}^{2}+2\left(\frac{\bar{l}_{\alpha}}{\alpha}\right)^{2}\|\Delta u(t)\|_{L^{2}(\Omega)}^{2} \\
& +2\left(\int_{0}^{t} L_{\alpha}^{2-r}(s) d s\right)\left(L_{\alpha}^{r} \square \Delta u\right)(t) \\
= & \left(\frac{1}{4}+2\left(\frac{\bar{l}_{\alpha}}{\alpha}\right)^{2}\right)\|\Delta u(t)\|_{L^{2}(\Omega)}^{2}+2\left(\int_{0}^{t} L_{\alpha}^{2-r}(s) d s\right)\left(L_{\alpha}^{r} \square \Delta u\right)(t),
\end{aligned}
$$


Multiplying (73) by $2 \eta\left(\xi^{\prime}(t)-\alpha \xi(t)\right)$ and using $\left|\frac{\xi^{\prime}(t)}{\xi(t)}\right| \leq L$, we get:

$$
\begin{aligned}
& 2 \eta\left(\xi^{\prime}(t)-\alpha \xi(t)\right) \int_{\Omega} \Delta u(t)\left(\int_{0}^{t} L_{\alpha}(t-s) \Delta u(s) d s\right) d x \\
\leq & \eta(L+\alpha)\left(\frac{1}{2}+4\left(\frac{\bar{l}_{\alpha}}{\alpha}\right)^{2}\right) \xi(t)\|\Delta u(t)\|_{L^{2}(\Omega)}^{2} \\
& +4 \eta(L+\alpha)\left(\int_{0}^{t} L_{\alpha}^{2-r}(s) d s\right) \xi(t)\left(L_{\alpha}^{r} \square \Delta u\right)(t) .
\end{aligned}
$$

Similarly, By using Young's inequality (for $\varepsilon=\delta_{3}$ ), we get:

$$
\begin{aligned}
& -2 \eta \xi(t)\left\{\int_{\Omega} \Delta u(t) \int_{0}^{t} l(t-s) \Delta u(s) d s d x\right\} \\
\leq & \frac{\eta}{2 \delta_{3}} \xi(t)(l \square \Delta u)(t)+2 \eta\left(1+\delta_{3}\right) \bar{l} \xi(t)\|\Delta u(t)\|_{L^{2}(\Omega)}^{2} .
\end{aligned}
$$

Making use of (70)-(72), (74) and (75) into (69), we get:

$$
\begin{aligned}
& \frac{d}{d t}\left\{V(t)+k_{1} \xi(0)\left(\int_{0}^{t}\left\|u_{s}(s)\right\|_{\rho+2}^{\rho+2} d s\right)+k_{2} \xi(0)\left(\int_{0}^{t}\|\Delta u(s)\|_{L^{2}(\Omega)}^{2} d s\right)\right. \\
& +k_{3} \xi(0)\left(\int_{0}^{t}\left\|\Delta u_{s}(s)\right\|_{L^{2}(\Omega)}^{2} d s\right)+k_{4} \xi(0)\left(\int_{0}^{t}\|u(s)\|_{\gamma}^{\gamma} d s\right) \\
& \left.+k_{5} \xi(0)\left(\int_{0}^{t}\left(L_{\alpha}^{r} \square \Delta u\right)(s) d s\right)\right\} \\
\leq & -\left\{k_{1}-\frac{\varepsilon L \delta_{1}[(\rho+2) e(0)] \frac{\rho}{2(\rho+2)}}{(\rho+1)^{2}}-\frac{\varepsilon}{(\rho+1)}\right\} \xi(t)\left\|u_{t}(t)\right\|_{\rho+2}^{\rho+2} \\
& -\left\{k_{2}+\varepsilon\left(\left(\xi_{0}-\bar{h}\right)-\frac{L C_{0} C_{p}}{2 \delta_{1}}-\frac{L}{2 \delta_{2}}-\frac{1}{4}\right)-\frac{\eta \bar{l}_{\alpha} L}{\alpha}-2 \eta \bar{l}_{\alpha}\right. \\
& \left.-\eta(L+\alpha)\left(\frac{1}{2}+4\left(\frac{\bar{l}_{\alpha}}{\alpha}\right)^{2}\right)-2 \eta\left(1+\delta_{3}\right) \bar{l}\right\} \xi(t)\|\Delta u(t)\|_{L^{2}(\Omega)}^{2} \\
& -\varepsilon \xi \xi \xi(t)\|\Delta u(t)\|_{L^{2}(\Omega)}^{2(\beta+1)}-\left\{k_{3}-\frac{\varepsilon L \delta_{2}}{2}-\varepsilon\right\} \xi(t)\left\|\Delta u_{t}(t)\right\|_{L^{2}(\Omega)}^{2}(\Omega) \\
& +\varepsilon \xi(t)\left(\int_{\Omega}|u|^{\gamma} \ln |u|^{k} d x\right)-k_{4} \xi(t)\|u(t)\|_{\gamma}^{\gamma} \\
& -\left\{\frac{1}{2}-\frac{\varepsilon}{\left(\xi_{0}-\bar{h}\right)}\left(\int_{0}^{t} h^{2-r}(s) d s\right)\right\} \xi(t)\left(h^{r} \square \Delta u\right)(t) \\
& -\left\{k_{5}-4 \eta(L+\alpha)\left(\int_{0}^{t} L_{\alpha}^{2-r}(s) d s\right)\right\} \xi(t)\left(L_{\alpha}^{r} \square \Delta u\right)(t) \\
& -\left\{\eta\left(1-\frac{1}{2 \delta_{3}}\right)-\frac{1}{2}\right\} \xi(t)(l \square \Delta u)(t) .
\end{aligned}
$$

Finally, we choose:

$$
\left\{\begin{array}{l}
\delta_{1}:=\frac{3 L C_{0} C_{p}}{2\left(\xi_{0}-\bar{h}\right)} \\
\delta_{2}:=\frac{3 L}{2\left(\xi_{0}-\bar{h}\right)} \\
\delta_{3}:=1
\end{array}\right.
$$


and:

$$
\left\{\begin{array}{l}
k_{1}:=\frac{\varepsilon}{\rho+1}\left(\frac{L \delta_{1}[(\rho+2) e(0)]^{\frac{\rho}{(\rho+2)}}}{(\rho+1)}+2\right) \\
k_{2}:=\frac{\varepsilon}{4}+(L+\alpha)\left(\frac{1}{2}+4\left(\frac{\bar{l}_{\alpha}}{\alpha}\right)^{2}\right)+4 \bar{l} \\
k_{3}:=\varepsilon\left(L \delta_{2}+2\right) \\
k_{5}:=8(L+\alpha)\left(\int_{0}^{t} L_{\alpha}^{2-r}(s) d s\right) \\
\eta:=1, \\
\varepsilon<\frac{\left(\tilde{\xi}_{0}-\bar{h}\right)}{4\left(\int_{0}^{t} h^{2-r}(s) d s\right)} .
\end{array}\right.
$$

Then, if $\bar{l}_{\alpha}<\frac{\alpha\left(\xi_{0}-\bar{h}\right)}{12 \alpha+6 L} \varepsilon$, we require from (76) that:

$$
\begin{aligned}
& \frac{d}{d t}\left\{V(t)+k_{1} \xi(0)\left(\int_{0}^{t}\left\|u_{s}(s)\right\|_{\rho+2}^{\rho+2} d s\right)+k_{2} \xi(0)\left(\int_{0}^{t}\|\Delta u(s)\|_{L^{2}(\Omega)}^{2} d s\right)\right. \\
& +k_{3} \xi(0)\left(\int_{0}^{t}\left\|\Delta u_{s}(s)\right\|_{L^{2}(\Omega)}^{2} d s\right)+k_{4} \xi(0)\left(\int_{0}^{t}\|u(s)\|_{\gamma}^{\gamma} d s\right) \\
& \left.+k_{5} \xi(0)\left(\int_{0}^{t}\left(L_{\alpha}^{r} \square \Delta u\right)(s) d s\right)\right\} \\
\leq & -\frac{k_{1}}{2} \xi(t)\left\|u_{t}(t)\right\|_{\rho+2}^{\rho+2}-\frac{\varepsilon\left(\xi_{0}-\bar{h}\right)}{6} \xi(t)\|\Delta u(t)\|_{L^{2}(\Omega)}^{2} \\
& -\varepsilon \xi \xi(t)\|\Delta u(t)\|_{L^{2}(\Omega)}^{2(\Omega+1)}-\frac{k_{3}}{2} \xi(t)\left\|\Delta u_{t}(t)\right\|_{L^{2}(\Omega)}^{2} \\
& +\varepsilon \xi(t)\left(\int_{\Omega}|u|^{\gamma} \ln |u|^{k} d x\right)-k_{4} \xi(t)\|u(t)\|_{\gamma}^{\gamma} \\
& -\frac{1}{4} \xi(t)(h \square \Delta u)(t)-\frac{k_{5}}{2} \xi(t)\left(L_{\alpha}^{r} \square \Delta u\right)(t) .
\end{aligned}
$$

By using (9) in (79), we get:

$$
\begin{aligned}
& \frac{d}{d t}\left\{V(t)+k_{1} \xi(0)\left(\int_{0}^{t}\left\|u_{s}(s)\right\|_{\rho+2}^{\rho+2} d s\right)\right. \\
& +k_{2} \xi(0)\left(\int_{0}^{t}\|\Delta u(s)\|_{L^{2}(\Omega)}^{2} d s\right) \\
& +k_{3} \xi(0)\left(\int_{0}^{t}\left\|\Delta u_{s}(s)\right\|_{L^{2}(\Omega)}^{2} d s\right)+k_{4} \xi(0)\left(\int_{0}^{t}\|u(s)\|_{\gamma}^{\gamma} d s\right) \\
& \left.+k_{5} \xi(0)\left(\int_{0}^{t}\left(L_{\alpha}^{r} \square \Delta u\right)(s) d s\right)\right\} \\
& \leq \quad-C_{3} \xi(t)\left\{E(t)+\left(h^{r} \square \Delta u\right)(t)+\left(L_{\alpha}^{r} \square \Delta u\right)(t)\right\}, \quad C_{3}>0 .
\end{aligned}
$$

Case 1. $r=1$.

From (80), this is written in the form:

$$
\begin{array}{ll} 
& \frac{d}{d t}\left\{V(t)+k_{1} \xi(0)\left(\int_{0}^{t}\left\|u_{s}(s)\right\|_{\rho+2}^{\rho+2} d s\right)+k_{2} \xi(0)\left(\int_{0}^{t}\|\Delta u(s)\|_{L^{2}(\Omega)}^{2} d s\right)\right. \\
& +k_{3} \xi(0)\left(\int_{0}^{t}\left\|\Delta u_{s}(s)\right\|_{L^{2}(\Omega)}^{2} d s\right)+k_{4} \xi(0)\left(\int_{0}^{t}\|u(s)\|_{\gamma}^{\gamma} d s\right) \\
& \left.+k_{5} \xi(0)\left(\int_{0}^{t}\left(L_{\alpha}^{r} \square \Delta u\right)(s) d s\right)\right\} \\
\leq & -C_{3} \xi(t)\left\{E(t)+(h \square \Delta u)(t)+\left(L_{\alpha} \square \Delta u\right)(t)\right\}
\end{array}
$$


By virtue of Proposition 1 (the right-hand side inequality) in (81), we find for all $t \geq 0$ :

$$
\begin{aligned}
& \quad \frac{d}{d t}\left\{V(t)+k_{1} \xi(0)\left(\int_{0}^{t}\left\|u_{s}(s)\right\|_{\rho+2}^{\rho+2} d s\right)+k_{2} \xi(0)\left(\int_{0}^{t}\|\Delta u(s)\|_{L^{2}(\Omega)}^{2} d s\right)\right. \\
& +k_{3} \xi(0)\left(\int_{0}^{t}\left\|\Delta u_{s}(s)\right\|_{L^{2}(\Omega)}^{2} d s\right)+k_{4} \xi(0)\left(\int_{0}^{t}\|u(s)\|_{\gamma}^{\gamma} d s\right) \\
& \left.++k_{5} \xi(0)\left(\int_{0}^{t}\left(L_{\alpha}^{r} \square \Delta u\right)(s) d s\right)\right\} \\
& \leq \quad-\frac{C_{3}}{C_{2}} \xi(t)\left\{V(t)+k_{1} \xi(0)\left(\int_{0}^{t}\left\|u_{s}(s)\right\|_{\rho+2}^{\rho+2} d s\right)+k_{2} \xi(0)\left(\int_{0}^{t}\|\Delta u(s)\|_{L^{2}(\Omega)}^{2} d s\right)\right. \\
& +k_{3} \xi(0)\left(\int_{0}^{t}\left\|\Delta u_{s}(s)\right\|_{L^{2}(\Omega)}^{2} d s\right)+k_{4} \xi(0)\left(\int_{0}^{t}\|u(s)\|_{\gamma}^{\gamma} d s\right) \\
& \left.+k_{5} \xi(0)\left(\int_{0}^{t}\left(L_{\alpha}^{r} \square \Delta u\right)(s) d s\right)\right\} .
\end{aligned}
$$

By a simple integration in $(82)$ over $(0, t)$, we find:

$$
\begin{aligned}
& V(t)+k_{1} \xi(0)\left(\int_{0}^{t}\left\|u_{s}(s)\right\|_{\rho+2}^{\rho+2} d s\right)+k_{2} \xi(0)\left(\int_{0}^{t}\|\Delta u(s)\|_{L^{2}(\Omega)}^{2} d s\right) \\
& +k_{3} \xi(0)\left(\int_{0}^{t}\left\|\Delta u_{s}(s)\right\|_{L^{2}(\Omega)}^{2} d s\right)+k_{4} \xi(0)\left(\int_{0}^{t}\|u(s)\|_{\gamma}^{\gamma} d s\right) \\
& +k_{5} \xi(0)\left(\int_{0}^{t}\left(L_{\alpha}^{r} \square \Delta u\right)(s) d s\right) \\
& \leq V(0) e^{-\frac{C_{3}}{C_{2}}\left(\int_{0}^{t} \xi(s) d s\right)}, t \geq 0 .
\end{aligned}
$$

Notice that by our assumption $E(0)>0$ in the theorem, we have $V(0)>0$. Again, by Proposition 1 (the left-hand side inequality), we conclude the assertion of our theorem:

$$
E(t) \leq \frac{V(0)}{C_{1}} e^{-\frac{C_{3}}{C_{2}}\left(\int_{0}^{t} \xi(s) d s\right)}, \quad t \geq 0 .
$$

Case 2. $\mathbf{1}<r<\frac{3}{2}$. Therefore, Lemma 4 (for $\theta:=\tau$ and $\rho:=r$ ) and (A2) yield:

$$
\begin{aligned}
(h \square \Delta u)(t) & \leq \gamma_{1}\left(e(0) \int_{0}^{\infty} h^{1-\tau}(s) d s\right)^{\frac{r-1}{r-1+\tau}}\left(\left(h^{r} \square \Delta u\right)(t)\right)^{\frac{\tau}{r-1+\tau}} \\
& \leq \gamma_{2}\left(\left(h^{r} \square \Delta u\right)(t)\right)^{\frac{\tau}{r-1+\tau}} .
\end{aligned}
$$

Similarly, using Lemma 4 (for $\theta:=\tau$ and $\rho:=r$ ) and (30) yields:

$$
\left(L_{\alpha} \square \Delta u\right)(t) \leq \gamma_{2}^{\prime}\left(\left(L_{\alpha}^{r} \square \Delta u\right)(t)\right)^{\frac{\tau}{r-1+\tau}},
$$

for some $\gamma_{1}$ and $\gamma_{2}$ positive constants. Therefore, for any $r_{1}>1$, we arrive at:

$$
\begin{aligned}
& \left\{E(t)+(h \square \Delta u)(t)+\left(L_{\alpha} \square \Delta u\right)(t)\right\}^{r_{1}} \\
\leq & \gamma_{3} e^{r_{1}-1}(0)\left(\left\|u_{t}(t)\right\|_{L^{2}(\Omega)}^{2}+\|\Delta u(t)\|_{L^{2}(\Omega)}^{2}+\|u(t)\|_{\gamma+2}^{\gamma+2}\right) \\
& +\gamma_{4}((h \square \Delta u)(t))^{r_{1}}+\gamma_{4}\left(\left(L_{\alpha} \square \Delta u\right)(t)\right)^{r_{1}} \\
\leq & \gamma_{3} e^{r_{1}-1}(0)\left(\left\|u_{t}(t)\right\|_{L^{2}(\Omega)}^{2}+\|\Delta u(t)\|_{L^{2}(\Omega)}^{2}+\|u(t)\|_{\substack{\gamma+2 \\
\gamma+2}}^{\gamma+2}\right) \\
& +\gamma_{5}\left(\left(L_{\alpha}^{r} \square \Delta u\right)(t)\right)^{\frac{\tau r_{1}}{r-1+\tau}}+\gamma_{5}\left(\left(h^{r} \square \Delta u\right)(t)\right)^{r-1+\tau} .
\end{aligned}
$$


By choosing $\tau=\frac{1}{2}$ and $r_{1}=2 r-1\left(\right.$ hence $\left.\frac{\tau r_{1}}{r-1+\tau}=1\right)$, estimate (83) gives, for some $\gamma_{6}>0$,

$$
\begin{aligned}
& \left\{E(t)+(h \square \Delta u)(t)+\left(L_{\alpha} \square \Delta u\right)(t)\right\}^{r_{1}} \\
\leq & \gamma_{6}\left\{E(t)+\left(\left(h^{r} \square \Delta u\right)(t)\right)+\left(L_{\alpha}^{r} \square \Delta u\right)(t)\right\} .
\end{aligned}
$$

By combining (84) and (33) into (80), we obtain:

$$
\begin{aligned}
& \frac{d}{d t}\left\{V(t)+k_{1} \xi(0)\left(\int_{0}^{t}\left\|u_{s}(s)\right\|_{\rho+2}^{\rho+2} d s\right)+k_{2} \xi(0)\left(\int_{0}^{t}\|\Delta u(s)\|_{L^{2}(\Omega)}^{2} d s\right)\right. \\
& +k_{3} \xi(0)\left(\int_{0}^{t}\left\|\Delta u_{s}(s)\right\|_{L^{2}(\Omega)}^{2} d s\right)+k_{4} \xi(0)\left(\int_{0}^{t}\|u(s)\|_{\gamma}^{\gamma} d s\right) \\
& \left.+k_{5} \xi(0)\left(\int_{0}^{t}\left(L_{\alpha}^{r} \square \Delta u\right)(s) d s\right)\right\} \\
& \leq-\frac{C_{3}}{\gamma_{6}} \xi(t)\left\{E(t)+(h \square \Delta u)(t)+\left(L_{\alpha} \square \Delta u\right)(t)\right\}^{r_{1}} \\
& \leq-\frac{C_{3}}{\gamma_{6}\left(C_{2}\right)^{r_{1}}} \xi(t)\left\{V(t)+k_{1} \xi(0)\left(\int_{0}^{t}\left\|u_{s}(s)\right\|_{\rho+2}^{\rho+2} d s\right)\right. \\
& +k_{2} \xi(0)\left(\int_{0}^{t}\|\Delta u(s)\|_{L^{2}(\Omega)}^{2} d s\right)+k_{3} \xi(0)\left(\int_{0}^{t}\left\|\Delta u_{s}(s)\right\|_{L^{2}(\Omega)}^{2} d s\right) \\
& \left.+k_{4} \xi(0)\left(\int_{0}^{t}\|u(s)\|_{\gamma}^{\gamma} d s\right)+k_{5} \xi(0)\left(\int_{0}^{t}\left(L_{\alpha}^{r} \square \Delta u\right)(s) d s\right)\right\}^{r_{1}} \text {. }
\end{aligned}
$$

A simple integration of $(85)$ over $(0, t)$ leads to:

$$
\begin{aligned}
& \quad V(t)+k_{1} \xi(0)\left(\int_{0}^{t}\left\|u_{s}(s)\right\|_{\rho+2}^{\rho+2} d s\right)+k_{2} \xi(0)\left(\int_{0}^{t}\|\Delta u(s)\|_{L^{2}(\Omega)}^{2} d s\right) \\
& +k_{3} \xi(0)\left(\int_{0}^{t}\left\|\Delta u_{s}(s)\right\|_{L^{2}(\Omega)}^{2} d s\right)+k_{4} \xi(0)\left(\int_{0}^{t}\|u(s)\|_{\gamma}^{\gamma} d s\right) \\
& +k_{5} \xi(0)\left(\int_{0}^{t}\left(L_{\alpha}^{r} \square \Delta u\right)(s) d s\right) \\
& \leq C_{1}^{*}\left(1+\int_{0}^{t} \xi(s) d s\right)^{-\frac{1}{r_{1}-1}}, \quad \forall t \geq 0 .
\end{aligned}
$$

Therefore,

$$
\begin{aligned}
& \int_{0}^{\infty}\left\{V(t)+k_{1} \xi(0)\left(\int_{0}^{t}\left\|u_{s}(s)\right\|_{\rho+2}^{\rho+2} d s\right)+k_{2} \xi(0)\left(\int_{0}^{t}\|\Delta u(s)\|_{L^{2}(\Omega)}^{2} d s\right)\right. \\
& +k_{3} \xi(0)\left(\int_{0}^{t}\left\|\Delta u_{s}(s)\right\|_{L^{2}(\Omega)}^{2} d s\right)+k_{4} \xi(0)\left(\int_{0}^{t}\|u(s)\|_{\gamma}^{\gamma} d s\right) \\
& \left.+k_{5} \xi(0)\left(\int_{0}^{t}\left(L_{\alpha}^{r} \square \Delta u\right)(s) d s\right)\right\} d t \\
& \leq C_{1}^{*} \int_{0}^{\infty} \frac{1}{\left(1+\int_{0}^{t} \xi(s) d s\right) \frac{1}{r_{1}-1}} d t .
\end{aligned}
$$

Since $\frac{1}{r_{1}-1}>0$ and $\left(1+\int_{0}^{t} \xi(s) d s\right) \rightarrow+\infty$ as $t \rightarrow+\infty$, we get:

$$
\begin{aligned}
& \int_{0}^{\infty}\left\{V(t)+k_{1} \xi(0)\left(\int_{0}^{t}\left\|u_{s}(s)\right\|_{\rho+2}^{\rho+2} d s\right)+k_{2} \xi(0)\left(\int_{0}^{t}\|\Delta u(s)\|_{L^{2}(\Omega)}^{2} d s\right)\right. \\
& +k_{3} \xi(0)\left(\int_{0}^{t}\left\|\Delta u_{s}(s)\right\|_{L^{2}(\Omega)}^{2} d s\right)+k_{4} \xi(0)\left(\int_{0}^{t}\|u(s)\|_{\gamma}^{\gamma} d s\right) \\
& \left.+k_{5} \xi(0)\left(\int_{0}^{t}\left(L_{\alpha}^{r} \square \Delta u\right)(s) d s\right)\right\} d t \\
& <\infty .
\end{aligned}
$$


In addition, by using (7) in (86), we have:

$$
\begin{aligned}
& t\left\{V(t)+k_{1} \xi(0)\left(\int_{0}^{t}\left\|u_{s}(s)\right\|_{\rho+2}^{\rho+2} d s\right)+k_{2} \xi(0)\left(\int_{0}^{t}\|\Delta u(s)\|_{L^{2}(\Omega)}^{2} d s\right)\right. \\
& +k_{3} \xi(0)\left(\int_{0}^{t}\left\|\Delta u_{s}(s)\right\|_{L^{2}(\Omega)}^{2} d s\right)+k_{4} \xi(0)\left(\int_{0}^{t}\|u(s)\|_{\gamma}^{\gamma} d s\right) \\
& \left.+k_{5} \xi(0)\left(\int_{0}^{t}\left(L_{\alpha}^{r} \square \Delta u\right)(s) d s\right)\right\} \\
\leq & \frac{C_{1}^{*} t}{\left(1+\int_{0}^{t} \xi(s) d s\right)^{\frac{1}{r_{1}-1}}} \\
\leq & C_{r} .
\end{aligned}
$$

Therefore, we obtain:

$$
\begin{aligned}
& \sup _{t \geq 0} t\left\{V(t)+k_{1} \xi(0)\left(\int_{0}^{t}\left\|u_{s}(s)\right\|_{\rho+2}^{\rho+2} d s\right)+k_{2} \xi(0)\left(\int_{0}^{t}\|\Delta u(s)\|_{L^{2}(\Omega)}^{2} d s\right)\right. \\
& +k_{3} \xi(0)\left(\int_{0}^{t}\left\|\Delta u_{s}(s)\right\|_{L^{2}(\Omega)}^{2} d s\right)+k_{4} \xi(0)\left(\int_{0}^{t}\|u(s)\|_{\gamma}^{\gamma} d s\right) \\
& \left.+k_{5} \xi(0)\left(\int_{0}^{t}\left(L_{\alpha}^{r} \square \Delta u\right)(s) d s\right)\right\} \\
& <\infty .
\end{aligned}
$$

We use (87) and (89) to get:

$$
\begin{aligned}
& \int_{0}^{\infty}\left\{V(t)+k_{1} \xi(0)\left(\int_{0}^{t}\left\|u_{s}(s)\right\|_{\rho+2}^{\rho+2} d s\right)+k_{2} \xi(0)\left(\int_{0}^{t}\|\Delta u(s)\|_{L^{2}(\Omega)}^{2} d s\right)\right. \\
& +k_{3} \xi(0)\left(\int_{0}^{t}\left\|\Delta u_{s}(s)\right\|_{L^{2}(\Omega)}^{2} d s\right)+k_{4} \xi(0)\left(\int_{0}^{t}\|u(s)\|_{\gamma}^{\gamma} d s\right) \\
& \left.+k_{5} \xi(0)\left(\int_{0}^{t}\left(L_{\alpha}^{r} \square \Delta u\right)(s) d s\right)\right\} d t \\
& +\sup _{t \geq 0} t\left\{V(t)+k_{1} \xi(0)\left(\int_{0}^{t}\left\|u_{s}(s)\right\|_{\rho+2}^{\rho+2} d s\right)+k_{2} \xi(0)\left(\int_{0}^{t}\|\Delta u(s)\|_{L^{2}(\Omega)}^{2} d s\right)\right. \\
& +k_{3} \xi(0)\left(\int_{0}^{t}\left\|\Delta u_{s}(s)\right\|_{L^{2}(\Omega)}^{2} d s\right)+k_{4} \xi(0)\left(\int_{0}^{t}\|u(s)\|_{\gamma}^{\gamma} d s\right) \\
& \left.+k_{5} \xi(0)\left(\int_{0}^{t}\left(L_{\alpha}^{r} \square \Delta u\right)(s) d s\right)\right\} \\
& <\infty .
\end{aligned}
$$


Then, by using (90) and Lemma 5 (for $\rho:=r$ ), we have:

$$
\begin{aligned}
& (h \square \Delta u)(t) \\
\leq \quad & C_{2}^{*}\left(t\left\|u_{x}(x, t)\right\|_{H}^{2}+\int_{0}^{t}\left\|u_{x}(x, s)\right\|_{H}^{2} d s\right)^{\frac{r-1}{r}} \\
& \times\left(\int_{0}^{t} h^{r}(t-s)\|\Delta u(x, t)-\Delta u(x, s)\|_{H}^{2} d s\right) \\
\leq & C_{2}^{*}\left[t \left\{V(t)+k_{1} \xi(0)\left(\int_{0}^{t}\left\|u_{s}(s)\right\|_{\rho+2}^{\rho+2} d s\right)+k_{2} \xi(0)\left(\int_{0}^{t}\|\Delta u(s)\|_{L^{2}(\Omega)}^{2} d s\right)\right.\right. \\
& +k_{3} \xi(0)\left(\int_{0}^{t}\left\|\Delta u_{s}(s)\right\|_{L^{2}(\Omega)}^{2} d s\right)+k_{4} \xi(0)\left(\int_{0}^{t}\|u(s)\|_{\gamma}^{\gamma} d s\right) \\
& \left.+k_{5} \xi(0)\left(\int_{0}^{t}\left(L_{\alpha}^{r} \square \Delta u\right)(s) d s\right)\right\} \\
& +\int_{0}^{t}\left\{V(t)+k_{1} \xi(0)\left(\int_{0}^{t}\left\|u_{s}(s)\right\|_{\rho+2}^{\rho+2} d s\right)+k_{2} \xi(0)\left(\int_{0}^{t}\|\Delta u(s)\|_{L^{2}(\Omega)}^{2} d s\right)\right. \\
& +k_{3} \xi(0)\left(\int_{0}^{t}\left\|\Delta u_{s}(s)\right\|_{L^{2}(\Omega)}^{2} d s\right)+k_{4} \xi(0)\left(\int_{0}^{t}\|u(s)\|_{\gamma}^{\gamma} d s\right) \\
& \left.\left.+k_{5} \xi(0)\left(\int_{0}^{t}\left(L_{\alpha}^{r} \square \Delta u\right)(s) d s\right)\right\} d t\right] \frac{r-1}{r}\left(\left(h^{r} \square \Delta u\right)(t) \frac{1}{r}\right. \\
\leq & C_{3}^{*}\left(\left(h^{r} \square \Delta u\right)(t)\right)^{\frac{1}{r},}
\end{aligned}
$$

which implies that:

$$
\left(h^{r} \square \Delta u\right)(t) \geq C_{4}^{*}((h \square \Delta u)(t))^{r},
$$

Similarly, we get:

$$
\left(L_{\alpha}^{r} \square \Delta u\right)(t) \geq C_{5}^{*}\left(\left(L_{\alpha} \square \Delta u\right)(t)\right)^{r},
$$

for some constant $C_{4}^{*}>0$ and $C_{5}^{*}>0$.

Consequently, a combination of (91) and (92) in (80) yields:

$$
\begin{aligned}
& \quad \frac{d}{d t}\left\{V(t)+k_{1} \xi(0)\left(\int_{0}^{t}\left\|u_{s}(s)\right\|_{\rho+2}^{\rho+2} d s\right)+k_{2} \xi(0)\left(\int_{0}^{t}\|\Delta u(s)\|_{L^{2}(\Omega)}^{2} d s\right)\right. \\
& +k_{3} \xi(0)\left(\int_{0}^{t}\left\|\Delta u_{s}(s)\right\|_{L^{2}(\Omega)}^{2} d s\right)+k_{4} \xi(0)\left(\int_{0}^{t}\|u(s)\|_{\gamma}^{\gamma} d s\right) \\
& \left.+k_{5} \xi(0)\left(\int_{0}^{t}\left(L_{\alpha}^{r} \square \Delta u\right)(s) d s\right)\right\} \\
& \leq- \\
& -C_{6} \xi(t)\left\{E(t)+((h \square \Delta u)(t))^{r}+\left(\left(L_{\alpha} \square \Delta u\right)(t)\right)^{r}\right\},
\end{aligned}
$$

for some constant $C_{6}>0$.

On the other hand, we can obtain:

$$
\begin{aligned}
& \left\{E(t)+(h \square \Delta u)(t)+\left(\left(L_{\alpha} \square \Delta u\right)(t)\right)\right\}^{r} \\
\leq & C_{7}\left\{E(t)+((h \square \Delta u)(t))^{r}+\left(\left(L_{\alpha} \square \Delta u\right)(t)\right)^{r}\right\},
\end{aligned}
$$


for all $t \geq 0$ and some constant $C_{7}>0$. Combining the last two inequalities and (33), we obtain:

$$
\begin{aligned}
& \frac{d}{d t}\left\{V(t)+k_{1} \xi(0)\left(\int_{0}^{t}\left\|u_{s}(s)\right\|_{\rho+2}^{\rho+2} d s\right)+k_{2} \xi(0)\left(\int_{0}^{t}\|\Delta u(s)\|_{L^{2}(\Omega)}^{2} d s\right)\right. \\
& +k_{3} \xi(0)\left(\int_{0}^{t}\left\|\Delta u_{s}(s)\right\|_{L^{2}(\Omega)}^{2} d s\right)+k_{4} \xi(0)\left(\int_{0}^{t}\|u(s)\|_{\gamma}^{\gamma} d s\right) \\
& \left.+k_{5} \xi(0)\left(\int_{0}^{t}\left(L_{\alpha}^{r} \square \Delta u\right)(s) d s\right)\right\} \\
\leq & -C_{6} \xi(t)\left\{E(t)+(h \square \Delta u)(t)+\left(\left(L_{\alpha} \square \Delta u\right)(t)\right)\right\}^{r} \\
\leq & -C_{7} \xi(t)\left\{V(t)+k_{1} \xi(0)\left(\int_{0}^{t}\left\|u_{s}(s)\right\|_{\rho+2}^{\rho+2} d s\right)+k_{2} \xi(0)\left(\int_{0}^{t}\|\Delta u(s)\|_{L^{2}(\Omega)}^{2} d s\right)\right. \\
& +k_{3} \xi(0)\left(\int_{0}^{t}\left\|\Delta u_{s}(s)\right\|_{L^{2}(\Omega)}^{2} d s\right)+k_{4} \xi(0)\left(\int_{0}^{t}\|u(s)\|_{\gamma}^{\gamma} d s\right) \\
& \left.+k_{5} \xi(0)\left(\int_{0}^{t}\left(L_{\alpha}^{r} \square \Delta u\right)(s) d s\right)\right\}^{r},
\end{aligned}
$$

for some constant $C_{7}>0$. A simple integration of $(92)$ over $(0, t)$ gives:

$$
\begin{aligned}
& V(t)+k_{1} \xi(0)\left(\int_{0}^{t}\left\|u_{s}(s)\right\|_{\rho+2}^{\rho+2} d s\right)+k_{2} \xi(0)\left(\int_{0}^{t}\|\Delta u(s)\|_{L^{2}(\Omega)}^{2} d s\right) \\
& +k_{3} \xi(0)\left(\int_{0}^{t}\left\|\Delta u_{s}(s)\right\|_{L^{2}(\Omega)}^{2} d s\right)+k_{4} \xi(0)\left(\int_{0}^{t}\|u(s)\|_{\gamma}^{\gamma} d s\right) \\
& +k_{5} \xi(0)\left(\int_{0}^{t}\left(L_{\alpha}^{r} \square \Delta u\right)(s) d s\right) \\
& \leq C_{8}\left(1+\int_{0}^{t} \xi(s) d s\right)^{-\frac{1}{r-1}}, \quad \forall t \geq 0 .
\end{aligned}
$$

Again by Proposition 1 (the left-hand side of the inequality), we conclude with the assertion of our theorem:

$$
E(t) \leq C\left(1+\int_{0}^{t} \xi(s) d s\right)^{-\frac{1}{r-1}}, \quad \forall t \geq 0 .
$$

Therefore, (49) is obtained. This completes the proof.

\section{Conclusions}

Our outcomes are natural extensions from the recent preceding ones in $([7,8,24])$, where the authors dealt with a viscoelastic equation and the system in more general decaying kernels and gave some common decay results, from which the usual exponential and polynomial rates are not only general cases. Our decay rate obtained in the third section is less general then that obtained in $[7,8]$ with respect to the right-hand side defined as the logarithmic nonlinearity, which is familiar in physics, since it appears clearly natural in inflation cosmology and supersymmetric filed theories, quantum mechanics, and nuclear physics (see $[5,14])$. This sort of problem has many applications in several branches of physics such as nuclear physics, optics, and geophysics (see [3,15]). In future work, we will try to extend this study for the evolutionary case of the presented problem, but by using the semigroup theory.

Author Contributions: All authors contributed equally.

Acknowledgments: The first author gratefully acknowledges Qassim University, represented by the Deanship of Scientific Research, for the material support of this research under Number 3733-alrasscac-2018-1-14-S during the academic year 1439AH/2018.

Conflicts of Interest: The authors declare no conflict of interest. 


\section{References}

1. Cavalcanti, M.; Cavalcanti, V.N.D.; Soriano, J.A. Exponential decay for the solution of semi linear viscoelastic wave equations with localized damping. Electron. J. Differ. Equ. 2002, 2002, 1-14.

2. Cavalcanti, M.; Cavalcanti, V.N.D.; Martinez, P. General decay rate estimates for viscoelastic dissipative systems. Nonlinear Anal. 2008, 68, 177-193. [CrossRef]

3. Enqvist, K.; McDonald, J. Q-balls and baryogenesis in the MSSM. Phys. Lett. B 1998, 425, 309-321. [CrossRef]

4. Kirchhoff, G. Vorlesungen uber Mechanik; Tauber: Leipzig, Germany, 1883.

5. Gorka, P. Logarithmic quantum mechanics: Existence of the ground state. Found. Phys. Lett. 2006, 19, 591-601. [CrossRef]

6. $\mathrm{Mu}, \mathrm{C}$; $\mathrm{Ma}$, J. On a system of nonlinear wave equatioins with Balakrishnan-Taylor damping. Z. Angew. Math. Phys. 2014, 65, 91-113. [CrossRef]

7. Mesloub, F.; Boulaaras, S. General decay for a viscoelastic problem with not necessarily decreasing kernel. J. Appl. Math. Comput. 2018, 58, 647-665. [CrossRef]

8. Ouchenane, D.; Boulaaras, S.; Mesloub, F. General decay for a class of viscoelastic problem with not necessarily decreasing kernel. Appl. Anal. 2018. [CrossRef]

9. Medjden, M.; Tatar, N.E. Asymptotic behavior for a viscoelastic problem with not necessarily decreasing kernel. Appl. Math. Comput. 2005, 167, 1221-1235. [CrossRef]

10. Boumaza, N.; Boulaaras, S. General Decay for Kirchhoff Type in Viscoelasticity with Not Necessarily Decreasing Kernel; John Wiley \& Sons, Ltd.: New York, NY, USA, 2018; doi:10.1002/mma.5117.

11. Zara, A.; Tatar, N.E. Global existence and polynomial decay for a problem with Balakrishnan-Taylor damping. Arch. Math. 2010, 46, 157-176.

12. Alabau-Boussouira, F.; Cannarsa, P. A general method for proving sharp energy decay rates for memory-dissipative evolution equations. C. R. Math. Acad. Sci. Paris Ser. I 2009, 347, 867-872. [CrossRef]

13. Bass, R.W.; Zes, D. Spillover nonlinearity, and flexible structures. In Proceedings of the Fourth NASA Workshop on Computational Control of Flexible Aerospace Systems, Williamsburg, VA, USA, 11-13 July 1991; pp. 1-14.

14. Bartkowski, K.; Gorka, P. One-dimensional Klein-Gordon equation with logarithmic nonlinearities. J. Phys. A 2008, 41, 355201. [CrossRef]

15. Barrow, J.; Parsons, P. Inflationary models with logarithmic potentials. Phys. Rev. D 1995, 52, 5576-5587. [CrossRef]

16. Cavalcanti, M.; Filho, V.N.D.; Cavalcanti, J.S.P.; Soriano, J.A. Existence and uniform decay rates for viscoelastic problems with nonlinear boundary damping. Differ. Integr. Equ. 2001, 14, 85-116.

17. Cavalcanti, M.; Oquendo, H.P. Frictional versus viscoelastic damping in a semi linear wave equation. SIAM J. Control Optim. 2003, 42, 1310-132. [CrossRef]

18. Nakao, M. Decay of solutions of some nonlinear evolution Equation. J. Math. Anal. Appl. 1977, 60, 542-549. [CrossRef]

19. Ono, K. Global existence, decay, and blow-up of solutions for some mildly degenerate nonlinear Kirchhoff strings. J. Differ. Equ. 1997, 137, 273-301. [CrossRef]

20. Berrimi, S.; Messaoudi, S.A. Exponential decay for the solution of semilinear viscoelastic wave equations with localized damping. Electron. J. Differ. Equ. 2004, 2002, 1-10.

21. Tatar, N.E.; Zarai, A. Exponential stability and blow up for a problem with Balakrishnan-Taylor damping. Demonstr. Math. 2011, 44, 67-90. [CrossRef]

22. Liu, W. Exponential or polynomial decay of solutions to a viscoelastic equation with nonlinear localized damping. J. Appl. Math. Comput. 2010, 32, 59-68. [CrossRef]

23. Li, M.R.; Tsai, L.Y. Existence and nonexistence of global solutions of some systems of semilinear wave equations. Nonlinear Anal. 2013, 54, 1397-1415. [CrossRef]

24. Zara, A.; Draifia, A.; Boulaaras, S. Blow up of solutions for a system of nonlocal singular viscoelatic equations. Appl. Anal. 2017. [CrossRef]

(C) 2019 by the authors. Licensee MDPI, Basel, Switzerland. This article is an open access article distributed under the terms and conditions of the Creative Commons Attribution (CC BY) license (http:/ / creativecommons.org/licenses/by/4.0/). 